\title{
Short- and medium-term impacts of strict anti-contagion policies on non-COVID-19 mortality in China
}

\author{
Jinlei Qi $\odot{ }^{1,9}$, Dandan Zhang $\oplus^{2,3,9}$, Xiang Zhang $\oplus^{2}$, Tanakao Takana $\oplus^{4}$, Yuhang Pan $\oplus^{5}$, Peng Yin', \\ Jiangmei Liu', Shuocen Liu', George F. Gao ${ }^{7,10} \bowtie$, Guojun He $\odot^{8,10 \bowtie}$ and Maigeng Zhou $\odot^{1,10 \bowtie ~}$
}

The effects of coronavirus disease-19 (COVID-19) public health policies on non-COVID-19-related mortality are unclear. Here, using death registries based on $\mathbf{3 0 0}$ million Chinese people and a difference-in-differences design, we find that China's strict anti-contagion policies during the COVID-19 pandemic significantly reduced non-COVID-19 mortality outside Wuhan (by 4.6\%). The health benefits persisted and became even greater after the measures were loosened: mortality was reduced by $12.5 \%$ in the medium term. Significant changes in people's behaviours (for example, wearing masks and practising social distancing) and reductions in air pollution and traffic accidents could have driven these results. We estimate that 54,000 lives could have been saved from non-COVID-19 causes during the 50 days of strict policies and 293,000 in the subsequent 115 days. The results suggest that virus countermeasures not only effectively controlled COVID-19 in China but also brought about unintended and substantial public health benefits.

B $y$ the end of 2020, COVID-19 had affected more than 219 countries and caused more than 1.7 million deaths worldwide $^{1}$. Facing this unprecedented crisis, different countries adopted various measures to mitigate its impacts, ranging from instances where governments imposed intense measures to curb the spread of the pandemic immediately after the outbreak, to instances where governments were reluctant to adopt measures to prevent the spread of the disease and explicitly resorted to herd immunity. Effective policies depend not only on the social preferences of people and the capacity of government but also on an accurate understanding of the costs and benefits of different COVID-19 countermeasures. However, relatively little is known about the broader impacts of these policies.

A key component of the evaluation of the welfare implications of anti-contagion policies is to examine their overall public health consequences in both the short and long term. Multiple studies have shown that strict social distancing and human mobility restrictions can effectively control the spread of COVID-19 and thus prevent deaths from the virus ${ }^{2-6}$. However, it remains unknown to researchers and policymakers how such interventions affect disease patterns and deaths from other causes. There are several competing arguments. For example, it has been suggested that measures such as lockdowns could harm overall population health in the short term because human mobility restrictions would reduce access to healthcare services, and business restrictions might lead to sharp economic disruption and massive layoffs ${ }^{7-13}$. In contrast, those who support strict anti-contagion policies (SAPs) argue that, without effective interventions, the pandemic would cause greater health damage in the long run because adverse economic damage could be more substantial and a larger number of COVID-19 patients might jeopardize healthcare provision. There is also speculation that virus containment policies might bring about unintended health benefits because they encourage health-protecting behaviours (for example, wearing face masks and practising better personal hygiene), diminish risks associated with business activities (for example, improving air quality and reducing work and traffic accidents) and reduce the transmission of other infectious diseases (for example, seasonal influenza) ${ }^{14-17}$. Thus, whether SAPs bring about additional health gains or losses is ultimately an empirical question.

Using death registries based on 300 million Chinese people, we examine how SAPs affect non-COVID-19-related mortality in both the short and medium term. We focus on China because this country was the first to mandate strict social distancing and SAPs to control the virus. Within a few weeks of COVID-19's being identified in Wuhan, a large number of cities enforced strict quarantines, implemented contact tracing, prohibited public gatherings, mandated social distancing and limited human mobility. These policies were often referred to as 'lockdowns' by the mass media but here we refer to them as SAPs. Many cities implemented these measures even though they had fewer than 100 confirmed cases (Extended Data Fig. 1). Our results will help researchers and policymakers worldwide understand the benefits and costs of SAPs during a pandemic, which will be valuable for current and future policy design.

The core of our empirical analysis uses the comprehensive and representative death records from China's Disease Surveillance Points (DSP) system, covering more than 324 million people in 605

\footnotetext{
'National Center for Chronic and Noncommunicable Disease Control and Prevention, Chinese Center for Disease Control and Prevention, Beijing, China. ${ }^{2}$ China Center for Economic Research, National School of Development, Peking University, Beijing, China. ${ }^{3}$ Institute for Global Health and Development, Peking University, Beijing, China. ${ }^{4}$ Division of Social Science, Hong Kong University of Science and Technology, Hong Kong SAR, China. ${ }^{5}$ Division of Environment and Sustainability, Hong Kong University of Science and Technology, Hong Kong SAR, China. ${ }^{6} \mathrm{HSBC}$ Business School, Peking University, Shenzhen, China. ${ }^{7}$ Chinese Center for Disease Control and Prevention, Beijing, China. ${ }^{8}$ Faculty of Business and Economics, University of Hong Kong, Hong Kong SAR, China. ${ }^{9}$ These authors contributed equally: Jinlei Qi, Dandan Zhang. ${ }^{10}$ These authors jointly supervised this work: George F. Gao, Guojun He, Maigeng Zhou.凶e-mail: gaofu@chinacdc.cn; gjhe@me.com; zhoumaigeng@ncncd.chinacdc.cn
} 
a

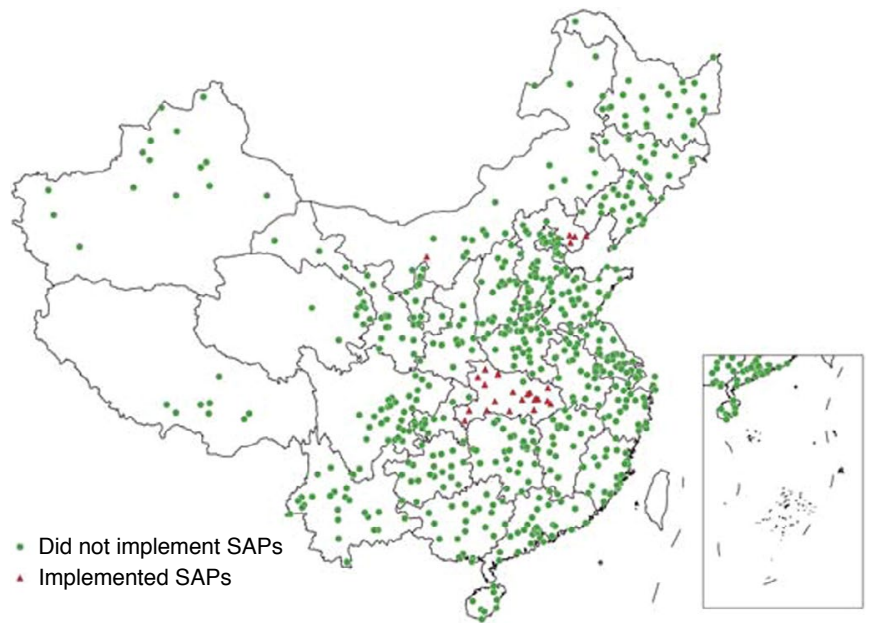

c

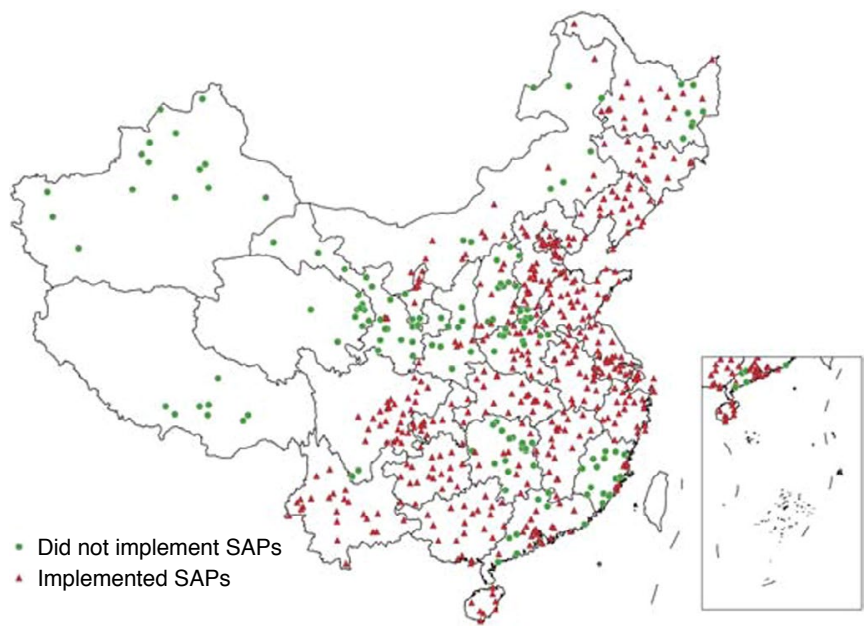

b

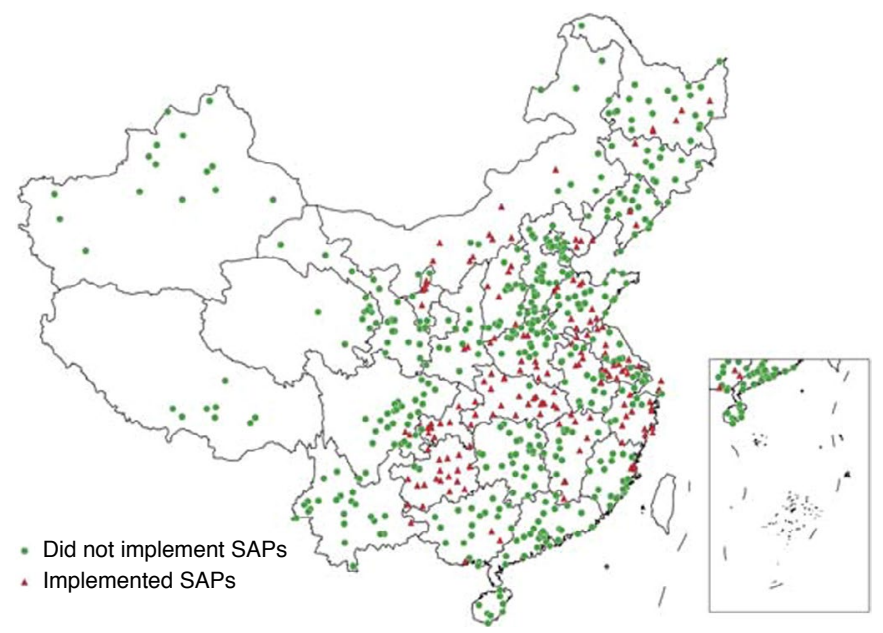

d

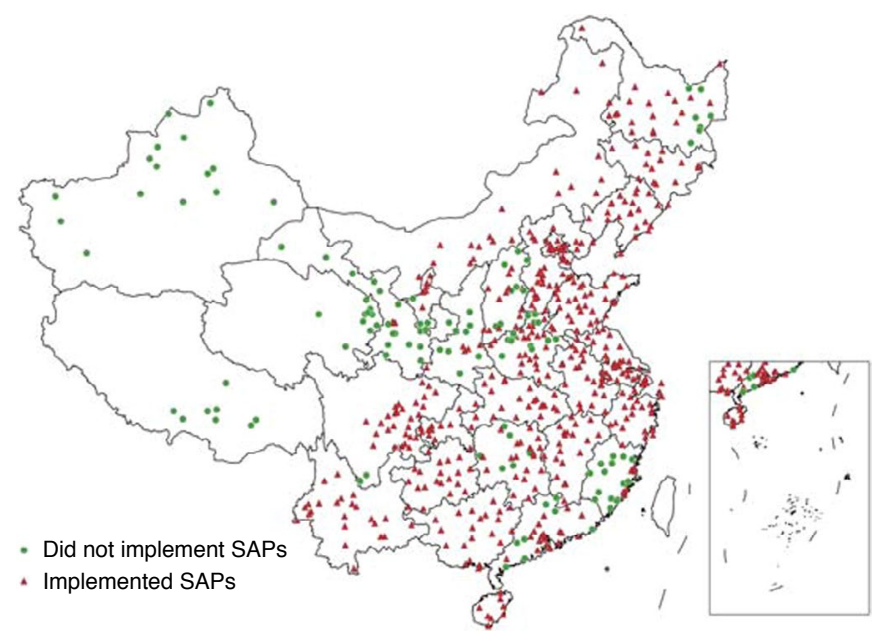

Fig. 1 Implementations of SAPs in the DSPs from January to February 2020. a-d, Maps showing the number of DSPs with SAPs implemented in different periods: 26 during 22-28 January (a), 160 during 29 January to 4 February (b), 460 during 5-11 February (c) and 486 during 12-18 February (d).

DSP districts/counties in 321 cities, accounting for $24.3 \%$ of the country's population ${ }^{18,19}$. Additionally, we collect information from various news media and government announcements on whether a city implemented SAPs ('lockdowns') (Fig. 1). Matching these datasets, we construct a daily DSP site-level panel dataset from 1 January to 31 July 2020. Our dataset includes $1,105,938$ death records in the DSP system reported until 28 September 2020 (Supplementary Table 1). Note that we exclude three DSPs in Wuhan from the baseline analysis because this city was the epicentre of the outbreak in China, and its death reporting process was disrupted due to insufficient medical resources during the COVID-19 outbreak ${ }^{15,20}$.

To quantify the impacts of SAPs on non-COVID-related mortality, we employ a difference-in-differences (DiD) approach, an econometric approach widely used to infer the causal effects of different interventions using observational data ${ }^{21}$. A key advantage of this approach is that it compares the policy effects relative to plausible counterfactuals. Specifically, the DiD model compares the changes in mortality between the DSPs with SAPs (treatment group) and the DSPs without such policies (control group) before and after the policies were enforced. In other words, the DSPs without SAPs (control group) serve as the counterfactual, mimicking what would have happened in the treated DSPs in the absence of SAPs.
Exploiting the staggered introduction of SAPs at the city and community level, we proceed in three steps. First, we estimate the short-term impacts of SAPs on the number of deaths from various causes, including cardiovascular diseases (CVDs), injury, acute lower respiratory infections (ALRIs), chronic lower respiratory infections (CLRIs), neoplasms and other causes during the SAP period. We can precisely identify the exact dates when SAPs were introduced for all the Chinese cities, but the dates of loosening SAPs are less clear. We thus use 8 April 2020 to define the post-policy period, when the 'lockdown' was lifted in Wuhan (the last city to 'reopen' in China). To ascertain that the estimates are not driven by confounding factors, we exploit several different model specifications: we use an event-study approach to assess whether the parallel trend assumption of the $\mathrm{DiD}$ model is reasonable, include various control variables, employ different matching estimators and regression weighting methods and focus on different subsamples to deal with different threats to accurate identification of cause and effect.

Second, we investigate whether the SAPs have long-lasting impacts on public health. We treat data from 8 April to 31 July 2020 as the post-SAP period and compare the mortality difference between the cities that had SAPs in place at some point and the cities that never implemented such policies, using the DiD design. 
We expect that the effects may have continued because most Chinese people continued to take preventative measures (for example, wearing masks and practising good hygiene) and business activities were not yet fully recovered even after COVID was under control.

Finally, we explore the heterogeneous impacts of SAPs across different cities. We hypothesize that results might differ along several dimensions: income levels, industrial structure, initial health status and initial pollution level. For example, it is plausible that, for cities with higher income and more abundant medical resources, the possible adverse effects of SAPs (such as reduced nutrition due to loss of income or loss of mobility to seek routine medical care) could be mitigated. In cities with worse population health (for example, higher mortality rate) and worse air quality, the potential health gains from SAPs (for example, less traffic and industrial activity, resulting in cleaner air) might be larger. The heterogeneity analyses provide insights into the channels through which the impacts of SAPs are manifested.

\section{Results}

Short-term impacts of SAPs. Figure 2 summarizes the baseline regression results obtained by fitting the $\mathrm{DiD}$ model of Equation (1) (see Table 1 for full results). Figure 2a reports the short-term effects on the number of non-COVID-related deaths, while Fig. $2 b$ reports the percentage change. Row 1 shows that overall the SAPs have a negative impact on non-COVID-19-related mortality. After human mobility was restricted, the DSP-level daily number of deaths decreased by $0.441(P<0.001,99 \% \mathrm{CI}-0.722$ to -0.160$)$, or $4.58 \%$, compared with the control group.

In rows (2) to (7), we separately examine the effects on different causes of death. We find that the number of deaths from CVDs was reduced by $5.85 \%$ (coefficient $-0.278, P<0.001,99 \% \mathrm{CI}-0.453$ to -0.103 ), deaths from injuries dropped by $6.28 \%$ (coefficient -0.035 , $P=0.015,95 \%$ CI -0.062 to -0.008 ) and deaths from ALRIs fell by $16.4 \%$ (coefficient $-0.022, P=0.012,95 \%$ CI -0.040 to -0.004 ) when SAPs were implemented. In contrast, the number of deaths from CLRIs, neoplasms and other diseases did not drop statistically significantly, although their coefficients are also negative.

We observe the largest drop in mortality caused by ALRIs. This is probably because the restrictions on mobility and rising awareness of personal hygiene reduced the likelihood of infection by other types of bacteria and viruses. We also observe a significant reduction in the number of deaths caused by CVDs, which could be caused by the improvement in air quality. Existing literature on the acute effects of air pollution suggests that elevated air pollution levels can substantially increase deaths from stroke, myocardial infarction and other types of $\mathrm{CVD}^{22-28}$. Consistent with previous studies, we find that SAPs indeed led to a sizeable improvement in air quality (Extended Data Fig. 2a), and deaths from all CVD subcategories were reduced (Extended Data Fig. 3a). Finally, because SAPs restricted production and social activities, work and traffic accidents also dropped significantly (subcategories in Extended Data Fig. 3b).

We complement these baseline results with the following additional analyses. A key assumption of the DiD model is that the treatment and the control group should follow parallel trends in the number of deaths in the absence of SAPs. We test this assumption using an event-study approach (Equation (3)) and find that the number of deaths in the treatment group does indeed parallel the number of deaths in the control group during the pre-treatment period (Fig. 3 and Supplementary Table 2). Note that the number of deaths from injuries (Fig. 3c) and ALRIs (Fig. 3d) started to decline 1 week prior to SAP implementation, implying that people were already taking precautionary actions 1 week before formal SAPs were implemented (Supplementary Note 1). Moreover, we find that our results are robust to additional controls, different regression weights, alternative definitions of SAPs and different

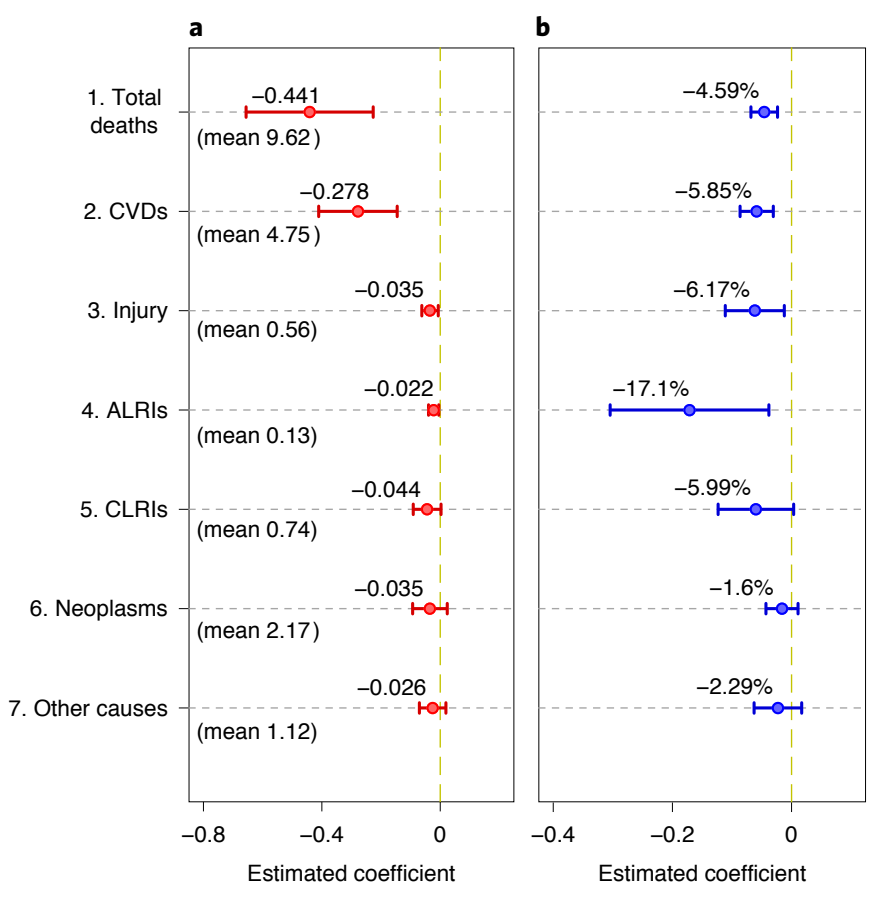

Fig. 2 | Short-term impacts of SAPs on non-COVID-19-related deaths. $\mathbf{a}, \mathbf{b}$, Impact of SAPs on number of non-COVID-19-related deaths (a) and percentage changes in deaths due to different causes (b). Dots and lines show point estimates and their $95 \%$ confidence interval, respectively. Each row in the figure represents a separate DiD regression using Equation (1). The number of observations for each regression is 58,996, covering 602 DSPs (excluding 3 DSPs in Wuhan). The outcome variable is the daily number of non-COVID-19-related deaths. We use mortality data from 1 January to 7 April 2020. The explanatory variable is a dummy indicating whether SAPs were implemented in a DSP's city on a particular date. We compute the percentage change by combining the estimates from $\mathbf{a}$ and the mean values for each cause of death. For example, $-4.58 \%$ in the first row of $\mathbf{b}$ is computed as $-0.441 / 9.62 \times 100 \%$. DSP and date fixed effects are included in each regression, and the standard errors are clustered at DSP level. Full results are presented in Table 1.

sampling strategies, ensuring that the strong relationship between SAPs and non-COVID-19-related deaths is not driven by a specific model choice (see Extended Data Fig. 4, Supplementary Note 2 and Supplementary Table 3 for details).

Medium-term impacts of SAPs. The effects of policy interventions can persist even after they are discontinued. In our setting, when SAPs were loosened in China, people were still encouraged to practise social distancing, keep good hygiene and reduce mobility (even though none of these was strictly mandated). Meanwhile, because economic activities had not yet fully recovered, the health risks imposed by air pollution and injuries remained low. Hence, we expect that the short-term health benefits of SAPs could be sustained, at least in the first few months after SAPs were lifted.

Figure 3 reports our findings (Equation (2) and Supplementary Table 4). The health benefits of SAPs persisted beyond the short term into the post-policy period (April 8 to July 31 ). In fact, if we compare the estimates, the reduction in overall mortality became even greater when virus containment measures were loosened. For example, the total number of non-COVID-19-related deaths was reduced by $12.5 \%$ (coefficient $-1,081, P<0.001,99 \%$ CI -1.650 to -0.512 ; Fig. 4 a) during the post-policy period, which is greater than the impact during the SAP period. Similar patterns can be observed 
Table 1 | Impacts of SAPs on deaths from different causes

\begin{tabular}{|c|c|c|c|c|c|c|c|}
\hline & (1) & (2) & (3) & (4) & (5) & (6) & (7) \\
\hline & Total no. of deaths & CVDs & Injury & ALRIs & CLRIs & Neoplasms & Other causes \\
\hline & Mean 9.620 & Mean 4.754 & Mean 0.557 & Mean 0.134 & Mean 0.738 & Mean 2.172 & Mean 1.195 \\
\hline & {$[0.109]$} & {$[0.068]$} & {$[0.014]$} & [0.009] & {$[0.024]$} & {$[0.030]$} & [0.023] \\
\hline DSP fixed effect & Yes & Yes & Yes & Yes & Yes & Yes & Yes \\
\hline Adjusted $R^{2}$ & 0.763 & 0.653 & 0.322 & 0.202 & 0.433 & 0.544 & 0.389 \\
\hline No. of DSP counties & 602 & 602 & 602 & 602 & 602 & 602 & 602 \\
\hline
\end{tabular}

Notes: Each cell in the table represents a separate DiD regression. All DSP districts/counties are included in the analysis except three from Wuhan. The outcome variable is the daily number of non-COVID 19-related deaths from the DSP districts/counties. We use mortality data from 1 January to 7 April 2020 for this analysis. The explanatory variable is a dummy indicating whether a city associated with a DSP had a SAP policy implemented on a particular date. Standard errors clustered at DSP level are reported below the estimates. ${ }^{\star} P<0.1$, ${ }^{\star \star} P<0.05,{ }^{\star \star \star} P<0.01$. Two-tailed $t$ tests are performed here and throughout the paper.
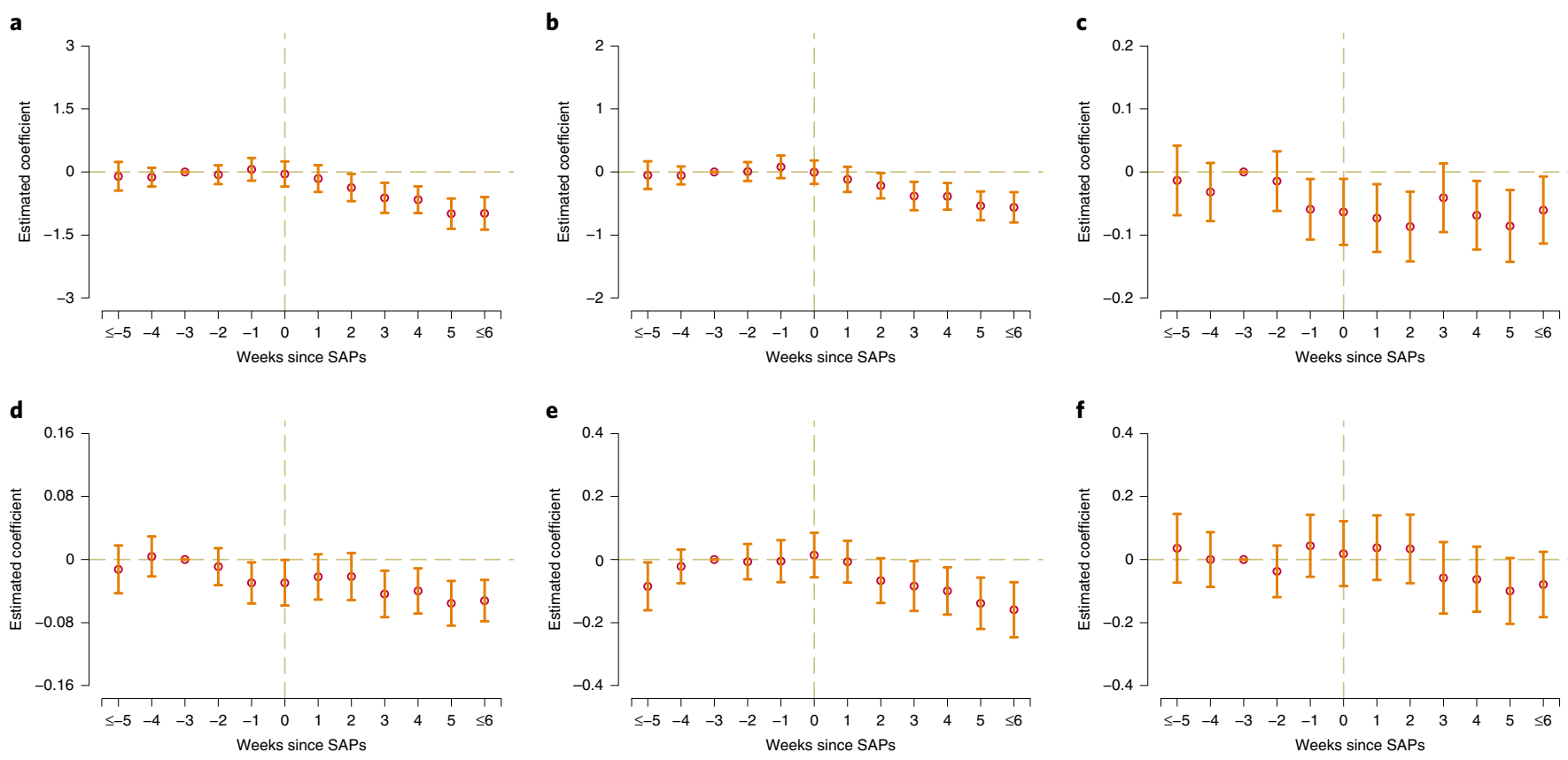

Fig. 3 | Event-study results on short-term effects of SAPs on non-COVID-19-related deaths. a-f, Separate regressions using the event-study approach (Equation (3)) for non-COVID-19-related deaths (a), and deaths due to CVDs (b), injury (c), ALRIs (d), CLRIs (e) and neoplasms (f). We include leads and lags of the start of SAPs in the regressions. The dummy variable indicating three weeks prior to implementation of SAPs $(k=-3)$ is omitted from the regressions. The estimated coefficients and their $95 \%$ confidence intervals are plotted. Vertical lines indicate the week when SAPs were implemented. The number of observations for each regression is 58,996, covering 602 DSPs (excluding 3 in Wuhan). We use mortality data from 1 January to 7 April 2020. DSP and date fixed effect are included in each regression, and standard errors are clustered at DSP level. Full results are presented in Supplementary Table 2.

across different causes of deaths, including CVDs by $15.2 \%$ (coefficient $-0.628, P<0.001,99 \%$ CI -0.968 to -0.288 ; Fig. $4 b$ ), injury by $8.4 \%$ (coefficient $-0.047, P=0.013,95 \% \mathrm{CI}-0.084$ to -0.010 ; Fig. 4 c), ALRIs by $39.6 \%$ (coefficient $-0.044, P<0.001,99 \%$ CI -0.070 to -0.018 ; Fig. 4 d), CLRIs by $17.7 \%$ (coefficient -0.107 , $P=0.008,99 \% \mathrm{CI}-0.210$ to -0.004 ; Fig. $4 \mathrm{e}$ ) and neoplasms by $6.0 \%$ (coefficient $-0.125, P=0.014,95 \%$ CI -0.225 to -0.026 ; Fig. 4 f). We describe the event-study results in Extended Data Fig. 5. These findings seem to suggest that the health benefits from better personal hygiene and improved air quality can accumulate rather than diminish, leading to greater health benefits ${ }^{29,30}$.
Heterogeneity of short-term impacts. In Fig. 5, we examine the heterogeneous impacts of SAPs on mortality (Equation (4) and Supplementary Table 5). We explore how cities' baseline characteristics, including income (measured by gross domestic product (GDP) per capita), healthcare resources (measured by number of hospital beds), air pollution levels (measured by air quality index), industrial structure (measured by employment share in manufacturing industries) and initial health status (measured by cause-specific mortality rate), are associated with the short-term health effects of SAPs. Note that the heterogeneity analyses only demonstrate factors that are correlated with a decline in mortality and do not have causal interpretations. 
a

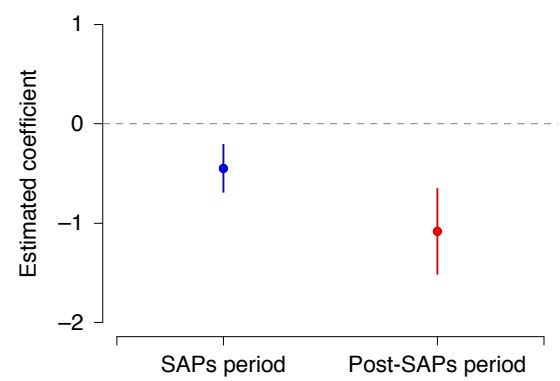

d

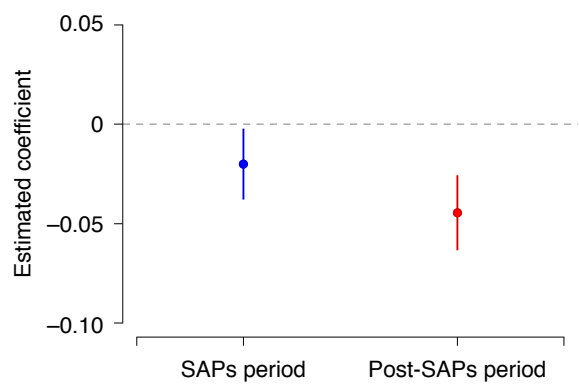

b

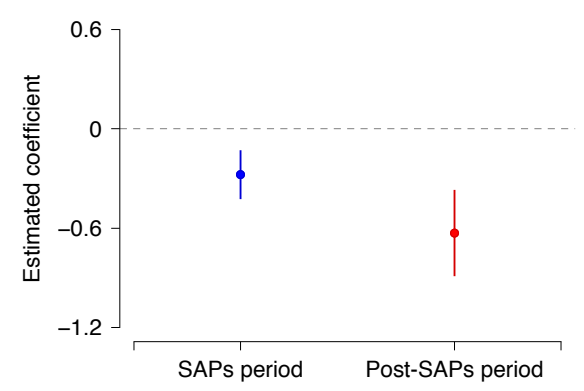

e

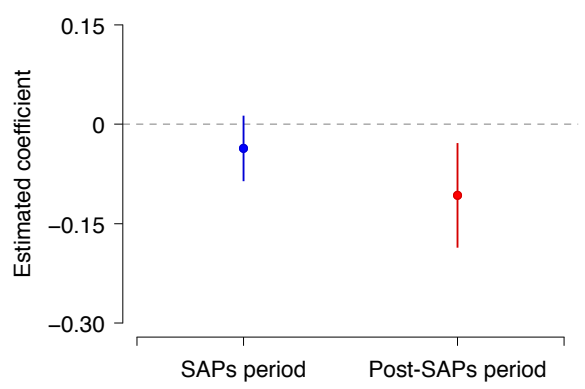

c
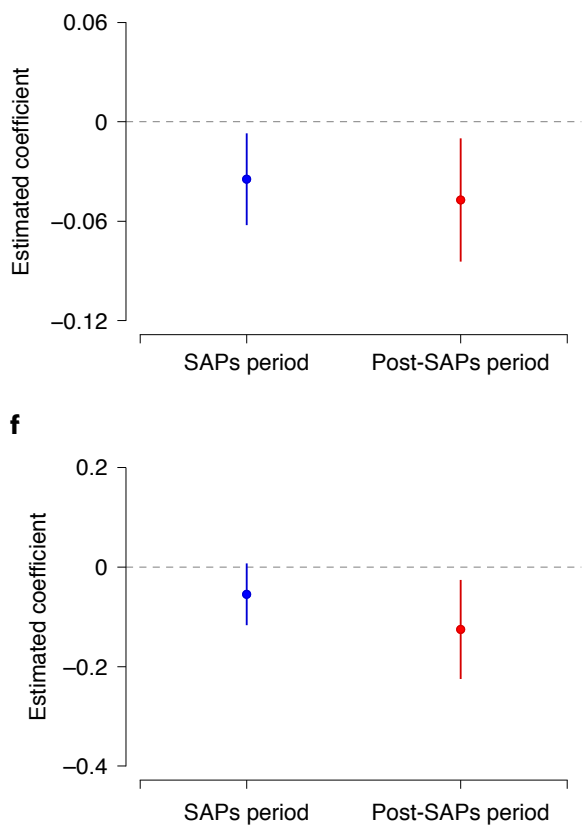

Fig. 4 | Short- and medium-term impacts of SAPs on deaths from different causes. a-f, Separate DiD regressions (Equation (2)) where the outcome variable is daily number of non-COVID-19 deaths (a) and deaths from CVDs (b), injury (c), ALRIs (d), CLRIs (e) and neoplasms (f). Dots and plots represent the point estimates and their 95\% confidence intervals for the short-term effects (blue, 1 January to 7 April) and medium-term effects (red, 8 April to 31 July). The number of observations for each regression is 58,996, covering 602 DSPs (excluding 3 in Wuhan). DSP and date fixed effects are included in each regression, and standard errors are clustered at DSP level. Full results are presented in Supplementary Table 4.

We observe two outstanding heterogeneous associations. First, the impacts of SAPs on non-COVID-19-related deaths (Fig. 5a), particularly from CVDs (Fig. 5c), ALRIs (Fig. 5d) and CLRIs (Fig. 5e), are greater when the DSP was initially more polluted. This is consistent with our finding that more polluted cities experienced greater air quality improvement during the pandemic (Extended Data Fig. 6), suggesting that air pollution can be an important factor driving the reduction in mortality during the pandemic. Second, cities with worse initial health status also demonstrated larger positive health impacts of SAPs (Fig. 5a-e). Our interpretation is that these cities were exposed to higher levels of health risks before the COVID-19 pandemic and the SAPs significantly mitigated such health risks.

GDP per capita and the availability of healthcare resources do not seem to play an important role. While some might conjecture that cities with lower income and scarce healthcare resources could experience more adverse effects of SAPs, we do not find strong evidence of this.

Finally, we investigate which age group(s) have the largest health gains from the SAPs. Extended Data Fig. 7 shows that the reduction in overall mortality is driven mostly by those aged above 65 years. For younger people, we observe fewer deaths caused by injuries. These results are consistent with our understanding that mortality risks are generally larger among the elderly, although young adults face higher risks of work and traffic accidents.

Estimation of averted non-COVID-19-related deaths. In Fig. 6, using the estimates in our analyses, we calculate the number of averted non-COVID-19 -related deaths in China due to the SAPs from 1 January to 7 April. During this period, 486 DSPs (80.7\%) eventually implemented SAPs, with an average duration of 50.4 days. Figure $6 \mathrm{a}$ plots the predicted number of non-COVID-19-related deaths (without SAPs) and the actual number of non-COVID-19-related deaths (with SAPs). Applying our estimates to all the Chinese cities that adopted SAPs during the study period, we calculate that the SAPs may have saved as many as 54,029 lives (Fig. 6b) during the policy implementation period. Looking at cause-specific effects, this reduction is mainly driven by fewer people dying from CVDs $(33,569)$. The SAPs also reduced the total number of people dying from injuries by 4,201 , from ALRIs by 2,431 , from CLRIs by 4,434 and from neoplasms by 6,613 .

During the post-policy period (115 days from 8 April to 31 July), the health benefits are greater. The estimated number of total averted deaths is 293,018 (173,731 from CVDs, 14,719 from injuries, 12,357 from ALRIs, 28,360 from CLRIs and 34,527 from neoplasms). In sum, during the entire study period ( 1 January to 31 July), SAPs may have averted 347,227 premature deaths in total in China.

\section{Discussion}

We investigated the consequences of community and city lockdowns in China in early 2020 on non-COVID-19-related mortality. We show that SAPs ('lockdowns') brought about unintended shortand medium-term health benefits. We observed fewer deaths from CVDs, traffic accidents, and ALRIs and CLRIs during the period of SAPs. These health benefits persisted into the post-lockdown period. Here, we discuss several implications of our findings.

Firstly, our findings demonstrate that China's SAPs unintendedly brought about sizeable health benefits. Given the increasingly heated debates regarding different counter-COVID-19 policy choices around the world, our results provide a benchmark to understand the broader health consequences of SAPs.

Second, our research also sheds light on directions for improving population health after the pandemic. For example, we observe a large decline in the number of deaths from infectious diseases (ALRIs): $16.4 \%$ in the short term and $39.6 \%$ in the medium term. This change is most likely driven by behavioural changes to avoid COVID-19 infections, which include wearing face masks, frequent hand sanitizing and washing and reducing physical interactions. 

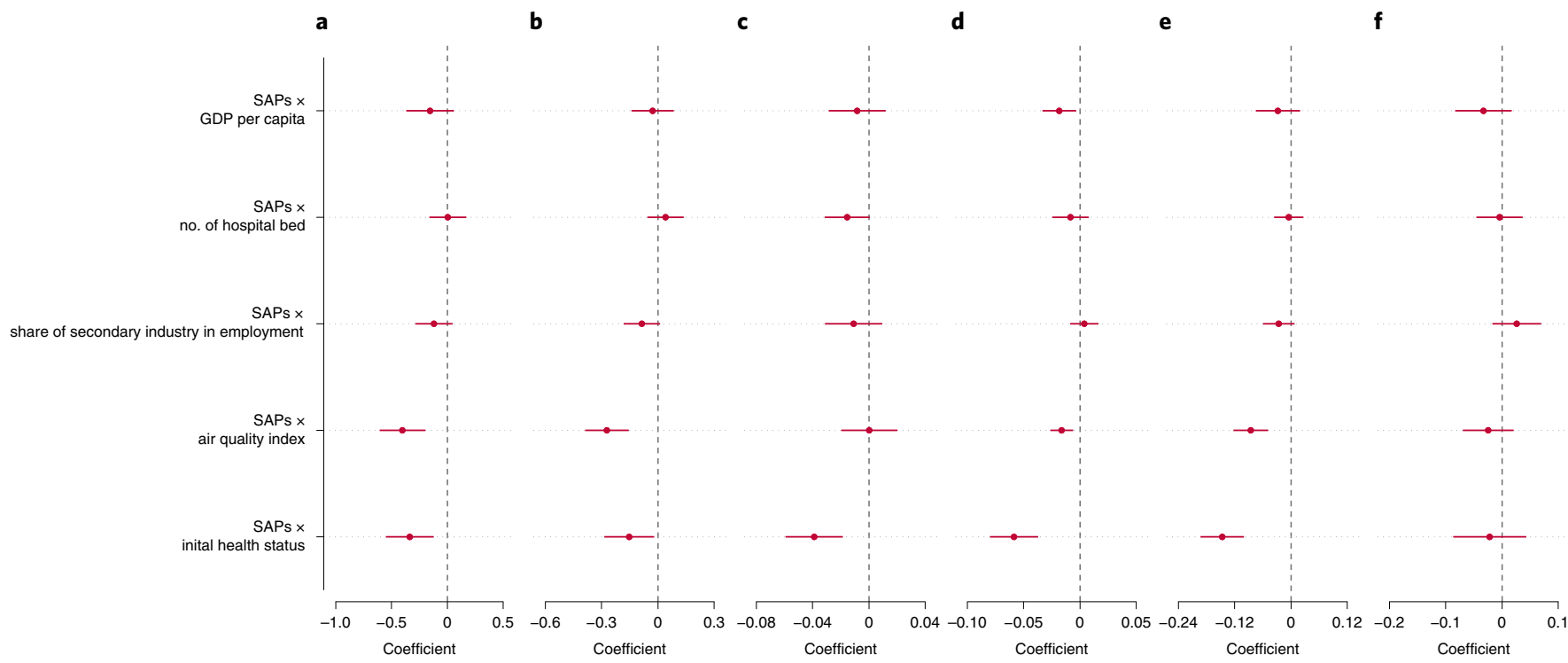

Fig. $\mathbf{5}$ | The heterogeneous impacts of SAPs on deaths. a-f, Separate DiD regressions where the outcome variable is daily number of non-COVID19-related deaths (a) and deaths due to CVDs (b), injury (c), ALRIs (d), CLRIs (e) and neoplasms (f). Dots and bars represent the point estimates and their $95 \%$ confidence intervals of the interaction terms between the SAP dummy and the heterogeneous dimensions (Equation (4)). We use GDP per capita, number of hospital beds per 1,000, share of employment in manufacturing, initial air quality index and initial mortality rate as heterogeneous dimensions. The details of these data are described in Methods. These variables are standardized to have a mean 0 and standard deviation of 1 so that the coefficients of interaction terms are comparable across variables. The number of observations for each regression is 58,996, covering 602 DSPs (excluding 3 in Wuhan). We use data from 1 January to 7 April 2020. DSP and date fixed effects are included in each regression, and standard errors are clustered at DSP level. Full results are presented in Supplementary Table 5.

We also observe a significant reduction in the number of deaths due to CVDs and speculate that this reduction is likely driven by a significant improvement in air quality. We provide evidence that cities with greater improvements in air quality also witnessed larger drops in non-COVID-19-related mortality during SAPs. Besides, less traffic, reduced occupational stress and changes in diet could also explain the public health benefits during the pandemic ${ }^{31-34}$. Among all these changes, we believe that some measures can be sustained at low cost (such as practising good personal hygiene) and will affect population health in the long term. Public health practitioners and governments need to identify effective ways to retain these health benefits after the pandemic.

Finally, our results stand in contrast to the suggestion that SAPs could increase mortality via increasing economic stress or limiting access to non-COVID-19-related medical care ${ }^{31}$. In fact, our analyses show positive health impacts of SAPs, at least in China and in the short and medium term.

We conclude by summarizing a few limitations of the research. First, due to the unavailability of data about personal daily activities and family care at the household level, we are unable to study the effect of SAPs on morbidity, especially for those who experienced non-COVID-related illness but avoided hospitals due to overcrowding or fear of contagion. Second, when investigating the medium-term impacts of SAPs, we only have 4 months (115 days) of data for the post-policy period. Although we observe that the health benefits of SAPs continued, it is unclear whether they will last longer. It is possible that, for example, after more people get vaccinated, their health behaviours will revert back to the pre-pandemic norm. It is also likely that, after economic production fully recovers, environmental quality will deteriorate and again threaten people's lives. Follow-up research should be conducted to examine the long-term consequences of SAPs when data become available. Third, there are concerns regarding the reliability of death records during the outbreak period because data reporting was sometimes disrupted and COVID-19 deaths may have been misclassified. However, we believe that these concerns will not affect our results: if misreporting were severe outside Wuhan, we would have expected to observe an unexplainable increase, rather than decrease, in the number of deaths from ALRIs or CLRIs, which have symptoms similar to COVID-19 (presumably, there were few cases of COVID-19 in the control group). Fourth, there may exist some spillover effects, in that neighbouring cities may also benefit from SAPs (through, for example, cleaner air in control cities). However, if this were true, we would under- rather than overestimate the health benefits of SAPs. Lastly, our study was limited to one country, China. Future research is warranted to explore the mortality changes in other countries during the pandemic.

\section{Methods}

Ethical approval. Data were analysed at an aggregate level, and no participants were contacted.

Data. Mortality data. We collected data on 1,105,938 deaths from 605 DSP districts/counties from 1 January to 31 July 2020 that were reported to the DSP system by 28 September 2020 . In our baseline analysis, we exclude three DSPs in Wuhan due to concerns that the data might not be reliable; a recent study showed that about 6,000 excess deaths (4,600 from pneumonia) were reported in the early stage of the pandemic in the city, while the number of COVID-19 deaths reported is 3,869 (refs. ${ }^{15,20}$ ). The causes of death are coded in accordance with the International Classification of Diseases-10th revision (ICD-10). We classified the main underlying causes of deaths into six categories: I00-I99 for CVDs, V01-Y89 for injuries, J09-J18 and J20-J22 for ALRIs, J40-J47 for CLRIs, C00-C97 for neoplasms, and remaining ICD-10 codes for all other causes. ALRIs include influenza, pneumonia and other ALRIs diseases caused by mycoplasmal, viral, bacterial and other infectious organisms. CLRIs include chronic bronchitis, emphysema, chronic obstructive pulmonary disease and asthma, which are not caused by infectious organisms. We further disaggregate deaths caused by CVDs and injuries into more specific diseases/causes. CVDs include stroke (I60-I62, I67 and I69), myocardial infarction (I20-I25) and other CVDs. Injuries include traffic accidents (V01-V04, V06, V09, V87, V89 and V99), suicide (X60-X84 and Y87) and other injuries. We also divide the daily number of deaths into three age groups (0-14, 15-64 and $\geq 65$ years). 


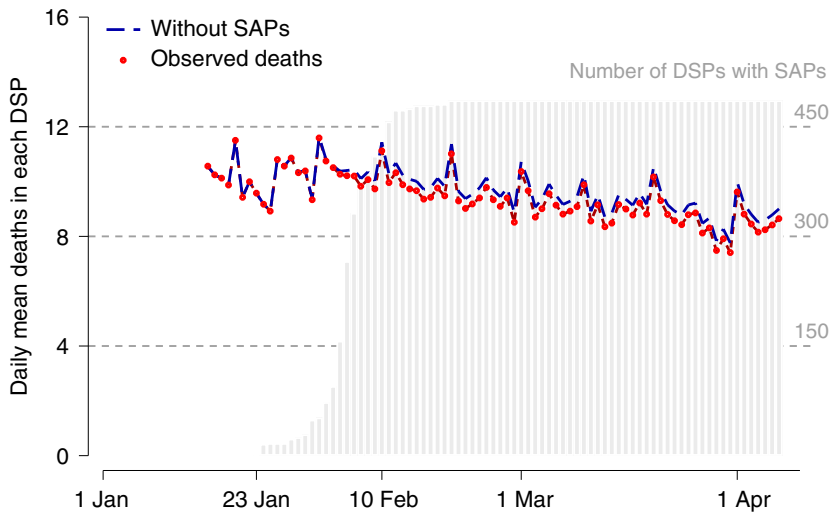

b

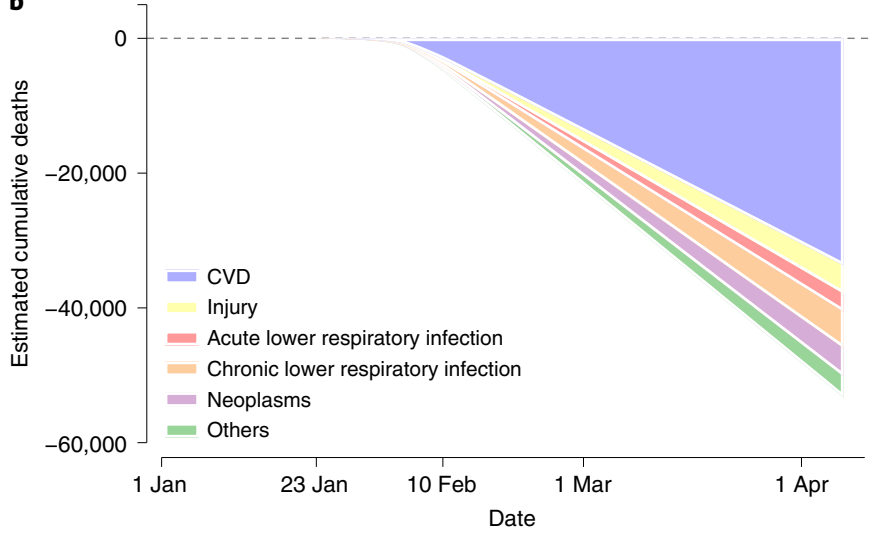

Fig. 6 | Estimated averted non-COVID-19-related deaths from SAPs.

Effect of SAPs on number of deaths relative to the counterfactual (without SAPs). a, Predicted average deaths per DSP per day with (red) and without (blue) SAPs from 1 January to 7 April. The difference between these two lines is regarded as the effect of SAPs, the red circles show observed deaths and the grey bars the cumulative percentage of DSPs with SAPs each day. b, Number of deaths from each disease that could be averted by SAPs in China, estimated using the entire nation's population in the cities with SAPS.

SAP data. We collected local governments' anti-contagion policies ('lockdowns' used by the Chinese government) city by city from news media and government announcements. Details are reported in Supplementary materials and methods. The evolution of different DSPs' SAPs is plotted in Fig. 1 and Extended Data Fig. 1. In Supplementary Table 6, we further provide a complete list of cities that adopted different SAPs on different dates. SAPs were gradually extended to different surveillance districts/counties between 23 January and 20 February. By the end of February, 486 out of 602 surveillance points had implemented SAPs. These SAPs were lifted in most cities between the end of March and the beginning of April, although exact dates of reopening were not publicly available. Wuhan, the last city to reopen in China, terminated its SAP policy on 8 April, so we treat 7 April as the last day of the SAP period.

Weather data. Weather variables include daily temperature, atmospheric pressure, relative humidity and wind speed. The data were obtained from the China Meteorology Administration. We aggregate station-level data to city-level data using the inverse squared distance (to city centres) as weights. Stations closer to the population centre are given higher weights so that city-level weather data can be representative of people dwelling in the city.

Air pollution data. We obtain air pollution data from the Ministry of Ecology and Environment. The original dataset included hourly air quality readings from over 1,600 monitoring stations covering 338 prefectural cities in China. We follow the same procedure to aggregate station-level air pollution data to the city level. As an omnibus measure of overall air quality, we use an air quality index and concentrations of $\mathrm{PM}_{2.5}$ (particles less than $2.5 \mu \mathrm{m}$ in diameter) and $\mathrm{PM}_{10}$ (particles less than $10 \mu \mathrm{m}$ in diameter) in our regressions.
Socio-economic conditions. We assemble the socio-economic data at the city or county level from the 2018 China City Statistical Yearbook and 2018 China County Statistical Yearbook, including GDP, population and number of hospital beds per 1,000 people. We also obtain data on the employment share of manufacturing and service industries using the 10\% sample of the 2015 1\% Population Sampling Survey in China.

Summary statistics. We report the summary statistics of the key variables for 602 DSPs in Supplementary Table 1. In panel A, we report the summary statistics of the DSP data. The average daily total number of deaths at the county level is 9.62 during our entire study period, with a standard deviation of 7.48. The leading cause of death during this period is CVDs, accounting for $49.4 \%$ of all deaths. The second leading cause of death is neoplasms (22.6\%), followed by CLRIs (7.7\%) and injuries $(5.8 \%)$. In panel B, we report the summary statistics of several other variables. The average air quality index during our study period is 69.98 , and the $\mathrm{PM}_{2.5}$ concentration is $46.91 \mathrm{\mu g} \mathrm{m}^{-3}$, five times higher than the World Health Organization standard $\left(10 \mu \mathrm{g} \mathrm{m}^{-3}\right.$ for annual mean, $25 \mu \mathrm{g} \mathrm{m} \mathrm{m}^{-3}$ for a daily mean). The average share of employment in the manufacturing industries was $24.2 \%$ as of 2015 .

Generalized DiD model. We use a generalized DiD model to estimate the impact of counter-COVID-19 measures on mortality. First, in our baseline regression, we estimate the relative change in the number of deaths between the treated and control DSPs using the following model:

$$
D_{i j t}=\alpha+\beta \times \operatorname{SAP}_{j t}+\lambda_{i}+\pi_{t}+\mathcal{E}_{i j t},
$$

where $D_{i j t}$ denotes the daily number of deaths in DSP $i$ in city $j$ on date $t$, and SAP $_{j t}$ is a dummy variable indicating whether DSP $i$ 's city $j$ implemented SAPs on date $t$. The SAP dummy equals 1 if either mobility restrictions across cities (city SAPs) or mobility restrictions within a city (community SAPs) were implemented, and 0 otherwise. Thus, the coefficient $\beta$ measures the average effect of three types of SAPs: city SAPs, community SAPs and both restrictions (city SAPs + community SAPs). Because most cities eventually implemented community SAPs, we also estimate the effects of community SAPs alone (Extended Data Fig. 4). $\lambda_{i}$ are DSP fixed effects, and $\pi_{t}$ indicate date fixed effects. $\mathcal{E}_{i j t}$ is the error term.

The county fixed effects, $\lambda_{i}$, which are a set of DSP-specific dummy variables, can control for time-invariant confounders specific to each DSP (for example, geographical conditions, industrial and economic structure, income and natural endowment). The date fixed effects, $\pi_{\mathrm{t}}$, are a set of dummy variables that account for shocks that are common to all DSPs on a given day, such as national holidays, macroeconomic conditions and the national trend in mortality. As both location and date fixed effects are included in the regression, the coefficient $\beta$ estimates the difference in the number of deaths between the treated (locked down) and the control cities before and after the implementation of SAPs. We also add a set of control variables to the regressions to check the robustness of the results (Extended Data Fig. 4).

The effects of SAPs may persist even after they were lifted. To investigate such medium-term effects of SAPs, we pool the data from 1 January to 31 July 2020, and fit the following equation:

$$
D_{i j t}=\alpha+\beta_{\mathrm{s}} \times \mathrm{SAP}_{j t}+\beta_{\mathrm{m}} \times \text { post } \mathrm{SAP}_{j t}+\lambda_{i}+\pi_{t}+\mathcal{E}_{i j t},
$$

where post $\mathrm{SAP}_{j t}$ is the interaction term between whether a city ever implemented SAPs ('lockdowns') and the post-policy period ( 8 April to 31 July). While $\beta_{\mathrm{s}}$ denotes the short-term impact of SAPs (during the policy implementation), $\beta_{\mathrm{m}}$ represents the medium-term impacts (post-policy implementation). Other variables are defined in the same way as in Equation (1). For the statistical test, standard errors are clustered at DSP level, and two-tailed $t$ tests are performed throughout the paper.

Event study. The underlying assumption for the $\mathrm{DiD}$ estimator is that treatment and control cities would have parallel trends in the number of deaths in the absence of SAPs. Even if the results show that mortality declined in the treatment counties after the implementation of SAPs, the results may not be driven by SAPs but by systematic differences in treatment and control cities. This assumption is untestable because we cannot observe the counterfactual: what would happen to the mortality levels in the locked-down counties if such policies were not enforced. Nevertheless, we can still examine the trends in mortality for both groups before the implementation of SAPs and investigate whether the two groups are comparable. To do so, we conduct an event study and fit the following equation:

$$
D_{i j t}=\alpha+\sum_{m=k, m \neq-3}^{M} \beta^{k} \times \mathrm{D} \_S A P_{j t, k}+\lambda_{i}+\pi_{t}+\mathcal{E}_{i j t}
$$

where D_SAP ${ }_{j t, k}$ are a set of dummy variables indicating the treatment status at different periods. Here, we put 7 days (1 week) into one bin (bin $m \in M$ ), so that the trend test is not affected by the high volatility of the daily number of deaths.

The dummy for $m=-3$ is omitted in Equation (3) so that the SAP effects are relative to the period 3 weeks before the launch of the policy. We use $m=-3$ as a reference because the effects of SAPs could appear before their implementation 
as some people might have started to drive less and adopt social distancing before the SAPs were announced by the government. The parameter of interest $\beta^{k}$ estimates the effect of SAPs $m$ weeks after their implementation. We include leads of the treatment dummy in the equation, testing whether the treatment affects the number of deaths before the launch of the policy. Intuitively, the coefficient $\beta^{k}$ measures the difference in the number of deaths between cities with SAPs and otherwise in period $k$ relative to the difference 2 weeks before SAP implementation. If the SAPs reduce mortality, $\beta^{k}$ would be negative when $k \geq-1$. If the pre-treatment trends are parallel, $\beta^{k}$ would be close to zero when $k \leq-2$.

Heterogeneity analysis. The impact of SAPs may not be evenly distributed across different regions. For instance, the effects could depend on cities' socio-economic status (for example, income, industrial structure and institutional capacities) and baseline health risks (for example, initial mortality rate and air pollution level). To estimate the heterogeneity, we fit the following equation:

$$
D_{i j t}=\alpha+\beta \times \operatorname{SAP}_{j t}+\sum_{h \in H} \gamma_{h} \times S A P_{j t} \times \operatorname{hvar}_{h}+\lambda_{i}+\pi_{t}+\mathcal{E}_{i j t},
$$

where $\mathrm{SAP}_{j t}$ hvar $_{h}$ is an interaction term between SAP status and variable $h$ $\left(\right.$ hvar $_{h}$ ) indicating the characteristics of city $j$. hvar $_{h}$ includes income (measured by GDP per capita in 2018), healthcare resources (measured by hospital beds per 1,000 people in 2018), industrial structure (measured by share of employment in manufacturing industries in 2015), air pollution levels (measured by average air quality index in 2019) and base health status (measured by each cause-specific mortality rate in 2019). We standardize hvar ${ }_{h}$ to have a mean of 0 and a standard deviation of 1 , so that we can compare the $\gamma_{h}$ across interaction terms. Note that $\gamma_{h}$ which is the parameter of interest, does not lend itself to a causal interpretation.

Back-of-the-envelope calculation. We can use the estimates from our DiD results to calculate the number of deaths averted in the entire country because our data are representative and cover around one-quarter of the Chinese population. To do so, we predict the number of deaths in two scenarios, that is, with and without SAPs. Taking the difference between these two predicted numbers of deaths, we can calculate the number of lives saved by the SAPs. We first predict the number of deaths with the SAPs in each DSP county/district each day by fitting the following model:

$$
\hat{D}_{i j t}=\hat{\alpha}+\hat{\beta} \times \mathrm{SAP}_{j t}+\hat{\lambda}_{\mathrm{i}}+\hat{\pi}_{t}
$$

where $\hat{D}_{i j t}$ denotes the deaths predicted with SAPs in each DSP county/district $i$ in city j. $\hat{\alpha}, \hat{\beta}, \hat{\lambda}_{\mathrm{i}}$, and $\hat{\pi}_{t}$ are the fitted values from Equation (1). In this function, the predicted deaths in each DSP, denoted by $\hat{D}_{i j t}$, can be affected by the SAP status (represented by $\mathrm{SAP}_{j t}$ ).

We then predict the counterfactual, that is, the number of deaths that would have occurred without the SAPs in any DSP, by fitting the following equation:

$$
\hat{D}_{i j t}(0)=\hat{\alpha}+\hat{\beta} \times \operatorname{SAP}_{j t}(0)+\hat{\lambda}_{\mathrm{i}}+\hat{\pi}_{t}
$$

where $\hat{D}_{i j t}(0)$ denotes the predicted averted deaths without any SAPs. $S A P_{j t}(0)$ always takes a value of 0 , so that this function is not affected by the policies. Taking the differences between $\hat{D}_{i j t}$ and $\hat{D}_{i j t}(0)$, we can calculate how many non-COVID19-related deaths were prevented as a result of the SAPs in each DSP each day.

Because the SAPs were implemented for 50.4 days on average, we estimate the following model to obtain the averted deaths in the whole country during our study period:

$$
\hat{D}_{\text {all }}=\frac{\text { Chpop }^{\mathrm{LD}}}{\operatorname{DSPpop}^{\mathrm{LD}}} \times \sum_{i \in I} \hat{D}_{i j t}-\hat{D}_{i j t}(0)
$$

where $\hat{D}_{\text {all }}$ denotes the averted deaths in the entire county during our study period, Chpop $^{\mathrm{LD}}$ denotes the total Chinese population in locked-down cities (around 1,161 million) and DSP pop ${ }^{\mathrm{LD}}$ represents the total population in locked-down DSPs counties/districts in our dataset (around 291 million in 486 DSPs). The difference between the scenarios with and without SAPs, denoted by $\hat{D}_{i j t}-\hat{D}_{i j t}(0)$, is totalled from 1 January to 7 April, which is our study period $(i \epsilon I)$. In our main text, we repeat these steps to estimate the averted deaths from each cause or disease to understand how many averted deaths can be attributed to different diseases/ causes. We can also estimate the number of averted deaths during the 115 days of the post-policy period ( 8 April to 31 July) by repeating the above process.

Reporting summary. Further information on research design is available in the Nature Research Reporting Summary linked to this article.

\section{Data availability}

Data used in this paper are available at https://github.com/yhecon/ Mortality_Covid_China.

\section{Code availability}

Code used in this paper are available at https://github.com/yhecon/

Mortality_Covid_China.
Received: 4 January 2021; Accepted: 23 July 2021;

Published online: 29 November 2021

\section{References}

1. Coronavirus Disease (COVID-19) Dashboard. World Health Organization https://www.who.int/emergencies/diseases/novel-coronavirus-2019.

2. Tian, H. et al. An investigation of transmission control measures during the first 50 days of the COVID-19 epidemic in China. Sci. (80-.) 368, 638-642 (2020).

3. Qiu, Y., Chen, X. \& Shi, W. Impacts of social and economic factors on the transmission of coronavirus disease 2019 (COVID-19) in China. J. Popul. Econ. 33, 1127-1172 (2020).

4. Chinazzi, M. et al. The effect of travel restrictions on the spread of the 2019 novel coronavirus (COVID-19) outbreak. Sci. (80-.). 368, 395-400 (2020).

5. Hsiang, S. et al. The effect of large-scale anti-contagion policies on the COVID-19 pandemic. Nature 584, 262-267 (2020).

6. Lai, S. et al. Effect of non-pharmaceutical interventions to contain COVID-19 in China. Nature 585, 410-413 (2020).

7. Baird, S., Friedman, J. \& Schady, N. Aggregate income shocks and infant mortality in the developing world. Rev. Econ. Stat. 93, 847-856 (2011).

8. Snyder, S. E. \& Evans, W. N. The effect of income on mortality: evidence from the social security notch. Rev. Econ. Stat. 88, 482-495 (2006).

9. Cutler, D. M., Knaul, F., Lozano, R., Méndez, O. \& Zurita, B. Financial crisis, health outcomes and ageing: Mexico in the 1980s and 1990s. J. Public Econ. 84, 279-303 (2002).

10. Adda, J., von Gaudecker, H. M. \& Banks, J. The impact of income shocks on health: evidence from cohort data. J. Eur. Econ. Assoc. 7, 1361-1399 (2009).

11. Falagas, M. E., Vouloumanou, E. K., Mavros, M. N. \& Karageorgopoulos, D. E. Economic crises and mortality: a review of the literature. Int. J. Clin. Pract. 63, 1128-1135 (2009).

12. Lundin, A., Lundberg, I., Hallsten, L., Ottosson, J. \& Hemmingsson, T. Unemployment and mortality - a longitudinal prospective study on selection and causation in 49321 Swedish middle-aged men. J. Epidemiol. Community Health 64, 22-28 (2010).

13. Vlachadis, N., Vrachnis, N., Ktenas, E., Vlachadi, M. \& Kornarou, E. Mortality and the economic crisis in Greece. Lancet 383, 691 (2014).

14. He, G., Pan, Y. \& Tanaka, T. The short-term impacts of COVID-19 lockdown on urban air pollution in China. Nat. Sustain. 3, 1005-1011 (2020).

15. Liu, J. et al. Excess mortality in Wuhan city and other parts of China during the three months of the Covid-19 outbreak: findings from nationwide mortality registries. BMJ https://doi.org/10.1136/bmj.n415 (2021)

16. Chen, K., Wang, M., Huang, C., Kinney, P. L. \& Anastas, P. T. Air pollution reduction and mortality benefit during the COVID-19 outbreak in China. The Lancet Planetary Health https://doi.org/10.1016/S2542-5196(20)30107-8 (2020).

17. Shilling, F. \& Waetjen, D. Special Report (Update): Impact of COVID19 Mitigation on Numbers and Costs of California Traffic Crashes. https:// roadecology.ucdavis.edu/files/content/projects/COVID_CHIPs_Impacts.pdf (2020).

18. Liu, S. et al. An integrated national mortality surveillance system for death registration and mortality surveillance, China. Bull. World Health Organ. 94, 46-57 (2016).

19. Yang, G. et al. Mortality registration and surveillance in China: history, current situation and challenges. Popul. Health Metr. 3, 3 (2005).

20. Wuhan's coronavirus death toll surges by $50 \%$ after China revision. The Wall Street Journal https://www.wsj.com/articles/ wuhans-coronavirus-death-toll-surges-by 50-after-china-reviews-data-11587110435 (2020).

21. Harris, D. \& Skeels, C. L. Mostly harmless econometrics: an empiricist's companion. Econ. Rec. 87, 350-352 (2011).

22. Chen, R. et al. Fine particulate air pollution and daily mortality: a nationwide analysis in 272 Chinese cities. Am. J. Respir. Crit. Care Med. https://doi. org/10.1164/rccm.201609-1862OC (2017).

23. Shah, A. S. V. et al. Short term exposure to air pollution and stroke: systematic review and meta-analysis. BMJ https://doi.org/10.1136/bmj.h1295 (2015)

24. Cheung, C. W., He, G. \& Pan, Y. Mitigating the air pollution effect? The remarkable decline in the pollution-mortality relationship in Hong Kong. J. Environ. Econ. Manag. 101, 102316 (2020).

25. He, G., Liu, T. \& Zhou, M. Straw burning, PM2.5, and death: evidence from China. J. Dev. Econ. 145, 102468 (2020).

26. Ebenstein, A., Fan, M., Greenstone, M., He, G. \& Zhou, M. New evidence on the impact of sustained exposure to air pollution on life expectancy from China's Huai River Policy. Proc. Natl Acad. Sci. U. S. A. 114, 10384-10389 (2017).

27. Yin, P. et al. Particulate air pollution and mortality in 38 of China's largest cities: time series analysis. BMJ https://doi.org/10.1136/bmj.j667 (2017). 
28. Mustafić, H. et al. Main air pollutants and myocardial infarction: a systematic review and meta-analysis. JAMA https://doi.org/10.1001/jama.2012.126 (2012).

29. Beverland, I. J. et al. A comparison of short-term and long-term air pollution exposure associations with mortality in two cohorts in Scotland. Environ. Health Perspect. https://doi.org/10.1289/ehp.1104509 (2012).

30. Anderson, M. L. As the wind blows: the effects of long-term exposure to air pollution on mortality. J. Eur. Econ. Assoc. 18, 1886-1927 (2020).

31. Tanaka, T. \& Okamoto, S. Increase in suicide following an initial decline during the COVID-19 pandemic in Japan. Nat. Hum. Behav. 5, 229-238 (2021).

32. Galasso, V. et al. Gender differences in COVID-19 attitudes and behavior: panel evidence from eight countries. Proc. Natl Acad. Sci. U. S. A. 117, 27285-27291 (2020).

33. Sheth, J. Impact of Covid-19 on consumer behavior: will the old habits return or die? J. Bus. Res. 117, 280-283 (2020).

34. Deschasaux-Tanguy, M. et al. Diet and physical activity during the coronavirus disease 2019 (COVID-19) lockdown (March-May 2020): results from the French NutriNet-Santé cohort study. Am. J. Clin. Nutr. https://doi. org/10.1093/ajcn/nqaa336 (2021)

\section{Acknowledgements}

The authors thank all the staff who work in the primary health facilities, hospitals and the Center for Disease Control and Prevention for death reporting at county/district, city, province and national levels. The authors thank Y. Qiu for sharing the community SAP data with us, and W. Wang and Y. Liu for providing excellent research assistance. The project is funded by the National Natural Science Foundation of China (82073675) (M.Z.), Peking University Research Grant (7100602966) (D.Z.), CIDEG Research Gran (2020CIDEGA02) of Tsinghua University (G.H.) and Anti-epidemic Fund 2.0 from the
HK Government (G.H.). The funders had no role in study design, data collection and analysis, decision to publish or preparation of the manuscript.

\section{Author contributions}

M.Z., G.H. and G.F.G. are joint senior co-authors. M.Z., G.H., D.Z. and J.Q. designed the project. J.Q., D.Z., X.Z. and S.L. curated data. D.Z., J.Q., X.Z., Y.P., P.Y., J.L. and T.T., analysed the data. M.Z., G.H, D.Z. and J.Q. interpreted the results. G.H., D.Z., J.Q, T.T. and Y.P. wrote the manuscript. G.H., G.F.G., J.Q., D.Z., T.T. and Y.P. edited the manuscript.

\section{Competing interests}

The authors declare no competing interests.

\section{Additional information}

Extended data is available for this paper at https://doi.org/10.1038/s41562-021-01189-3.

Supplementary information The online version contains supplementary material available at https://doi.org/10.1038/s41562-021-01189-3.

Correspondence and requests for materials should be addressed to George F. Gao, Guojun He or Maigeng Zhou.

Peer review information Nature Human Behaviour thanks Francesco Forastiere and the other, anonymous, reviewer(s) for their contribution to the peer review of this work.

Reprints and permissions information is available at www.nature.com/reprints. Publisher's note Springer Nature remains neutral with regard to jurisdictional claims in published maps and institutional affiliations.

(C) The Author(s), under exclusive licence to Springer Nature Limited 2021 
A. COVID-19 Confirmed, Active, Recovered, and Deaths

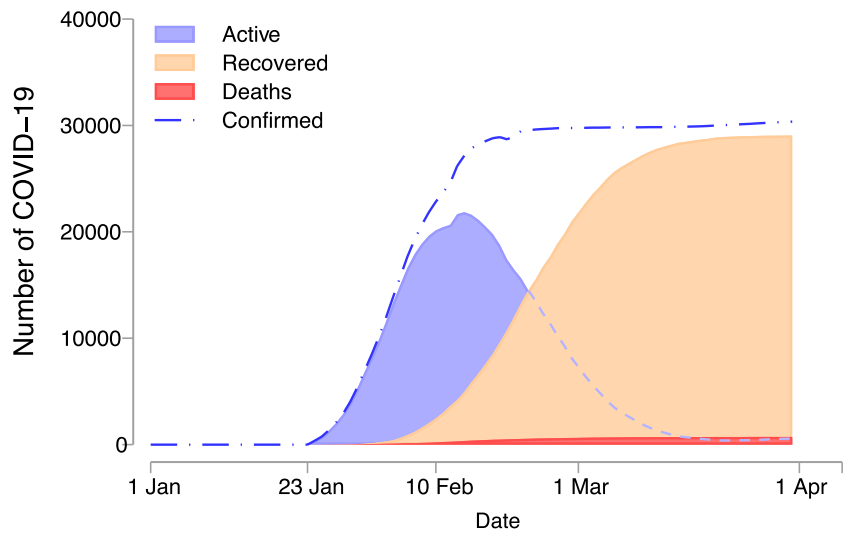

C. Timing of City/Community SAPs

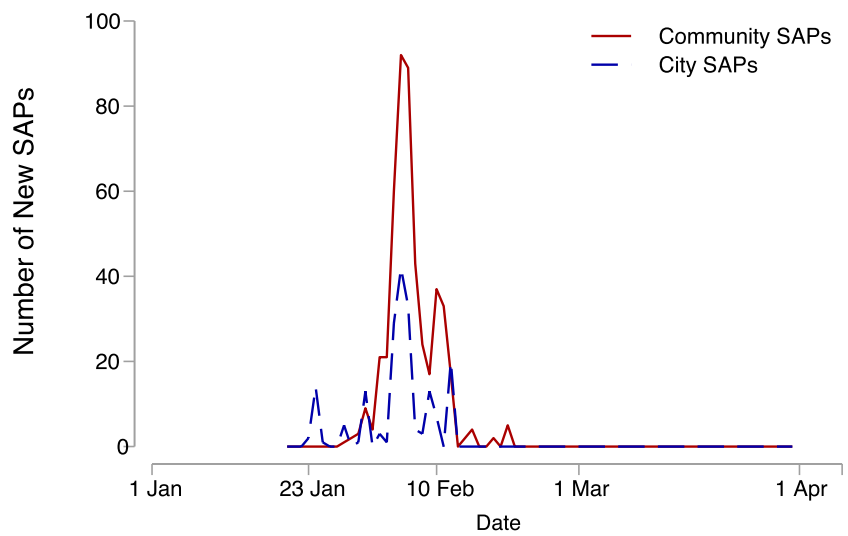

\section{B. Confirmed COVID-19 Cases in Each City}

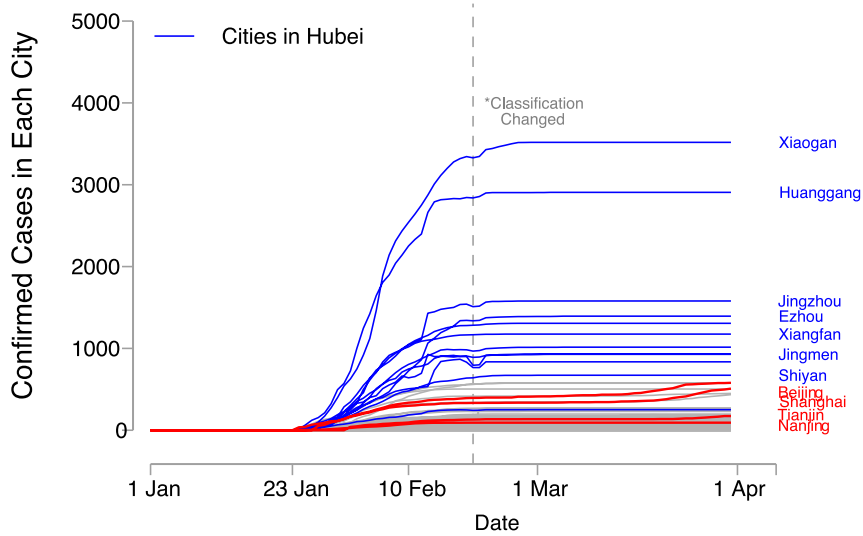

D. COVID-19 Before the City SAPS

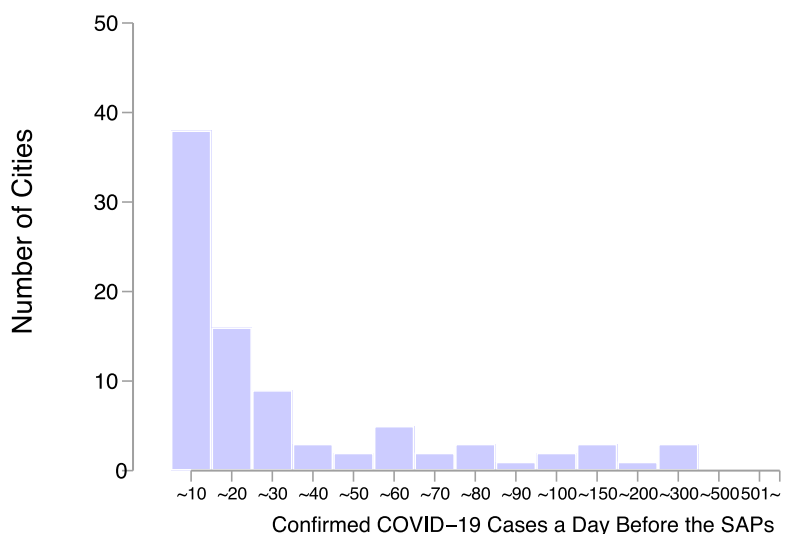

Extended Data Fig. 1 | COVID-19 and the SAPs outside of Wuhan in China. Panel A shows the evolution of the COVID-19, including the number of total confirmed cases, recovered cases, active cases, and deaths outside Wuhan in China. Panel B represents the confirmed COVID-19 cases in each city. The blue line denotes cities in Hubei province. Panel $C$ shows the timing of the start of the city/community SAPs. The red line represents the community SAPs that restrict human mobility within a city, and the blue line represents the city SAPs that restrict mobility across cities. Panel D describes the distribution of the number of confirmed cases a day before the implementation of SAPs. Because the confirmed COVID-19 cases are only available at the city level, the graph is based on city-level information. 
1. Air Quality Index
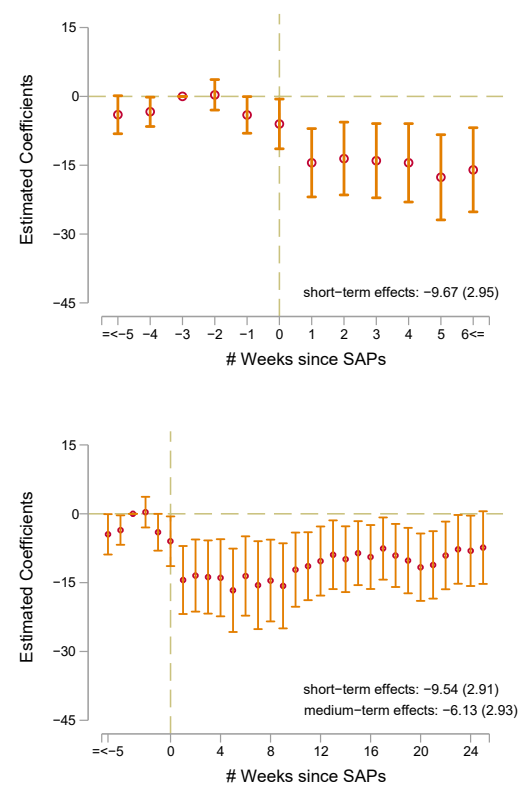

2. PM2.5
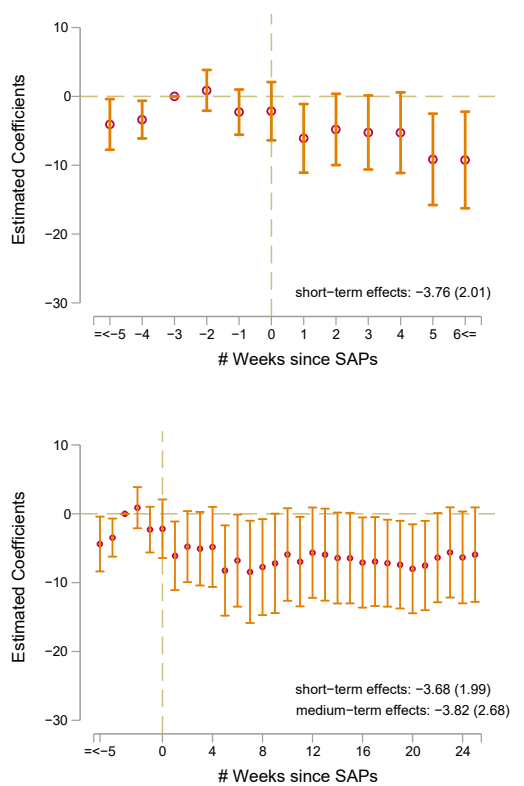

3. PM10
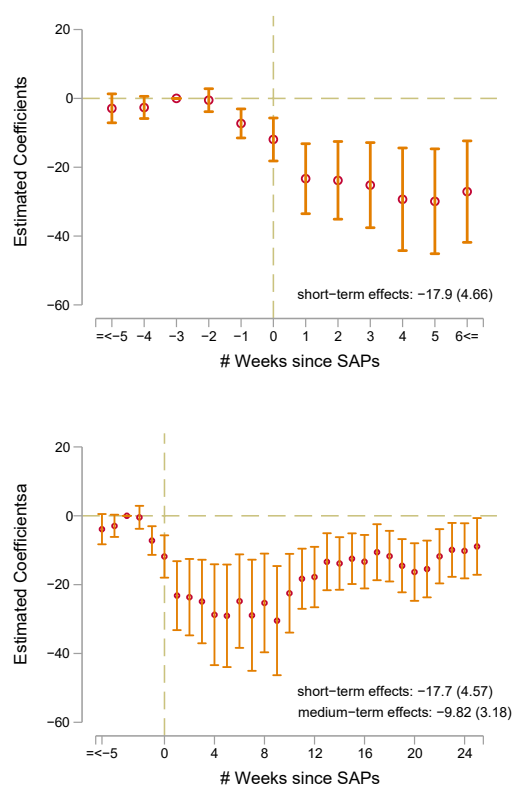

Extended Data Fig. 2 | The event-study results on the short- and medium-term effects of SAPs on air quality. Each figure represents a separate regression using the event-study approach (Equation 3). Panel A shows the short-term effects (January 1 to April 7) of SAPs on air quality, proxied by Air Quality Index (column 1), $\mathrm{PM}_{2.5}$ (2), and $\mathrm{PM}_{10}$ (3), while Panel B shows both the short- (January 1 to April 7) and medium-term effects (April 8 to July 31 ). We include leads and lags of the start of SAPs dummy in the regressions. The dummy variable indicating three weeks prior to the SAPs $(k=-3)$ is omitted from the regressions. The estimated coefficients and their $95 \%$ confidence intervals are plotted. The vertical lines refer to the week when SAPs are issued. The number of observations is 56,992 (A1), 56,986 (A2), 56,950 (A3), 123,864 (B1), 123,856 (B2),123,966 (B3) from 602 DSPs except for 3 DSPs in Wuhan. DSP fixed effect and date fixed effect are included in each regression, and the standard errors are clustered at the DSP level. 
A. CVD

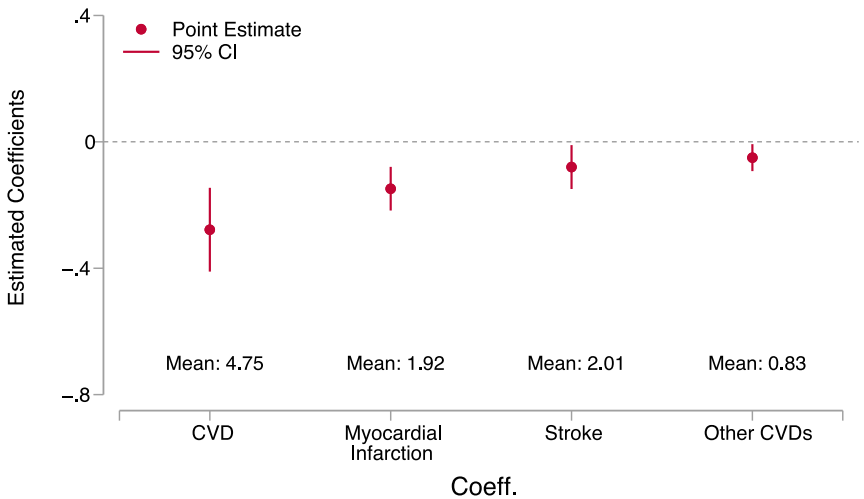

B. Injury

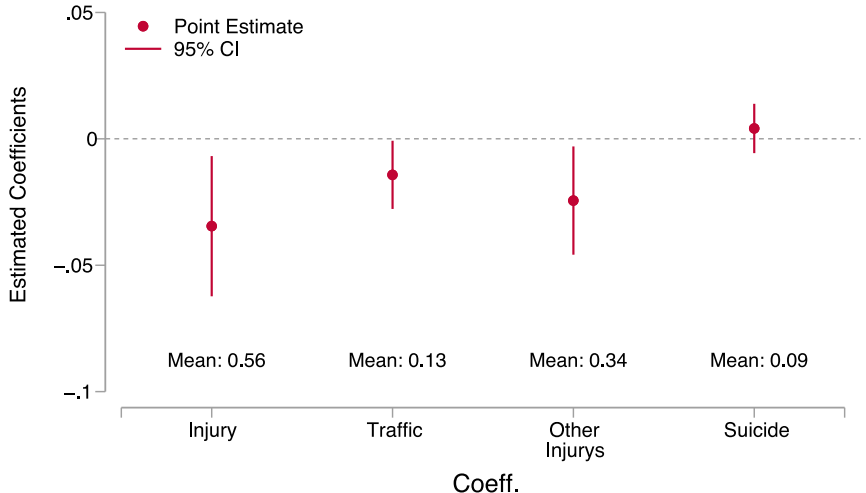

Extended Data Fig. 3 | The short-term impacts of SAPs on deaths from subcategories of CVD and injury. Each plot and bar in the figures represent a separate DiD regression (Equation 1) with red dots and the lines representing the point estimates and their $95 \%$ confidence intervals. Results report the effects of SAPs' impacts on the number of deaths (except for deaths from COVID-19) from subcategories of CVD (A), and injury (B). The number of observations for each regression is 58,996, covering 602 DSPs (3 DSPs in Wuhan are not included). We use mortality data from January 1 to April 7, 2020. The explanatory variable is a dummy indicating whether the DSP has implemented the SAPs on a particular date. DSP fixed effect and date fixed effect are included in each regression, and the standard errors are clustered at the DSP level. 


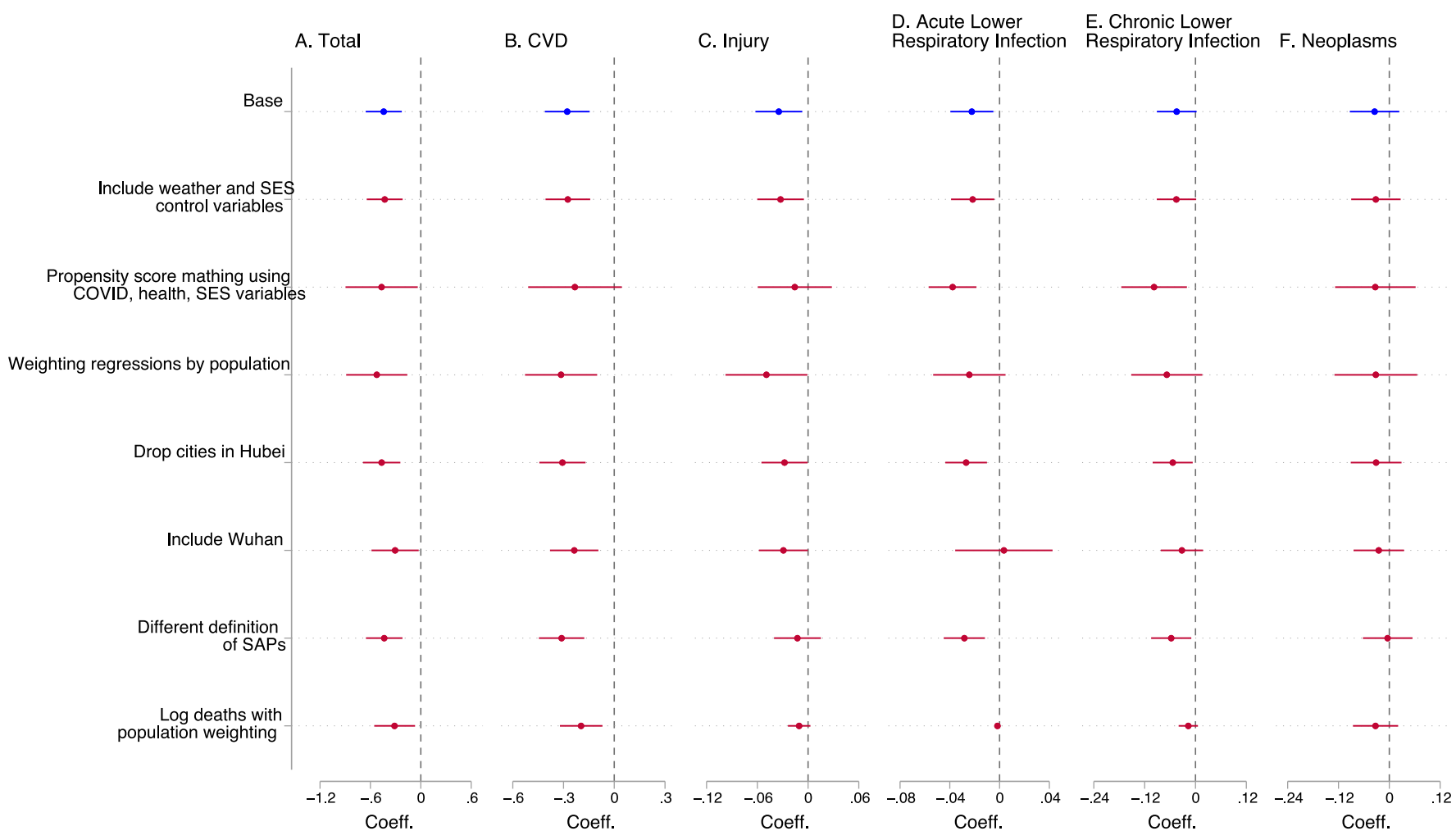

Extended Data Fig. 4 | Robustness check. Each plot and bar in the figures represent a separate DiD regression with plots and bars representing the point estimates and 95\% confidence interval. Results report the effects of SAPs on the number of deaths (except for deaths from COVID-19) from all-causes (A), CVD (B), injury (C), ALRI (D), CLRI (E), and neoplasms (F) using various model specifications. Row (1) describes the baseline estimates. Row (2) includes the time-varying weather variables and socio-economic status controls: interactions between time-invariant variables and a third-order polynomial function of time. Row (3) shows the results using propensity score matching + DiD, where we use the COVID-19 incidence (whether a city confirms at least one case and the first day of arrival), base mortality rate measured in 2019 (total, and each category), and socio-economic status (per capita GDP, number of hospital bed, share of secondary industry in GDP, base air quality index). Row (4) weights regressions using population as a weight. Row (5) includes 3 DSPs in Wuhan, while Row (6) drops 22 DSPs (3 DSPs in Wuhan and 19 DSPs in other cities) in Hubei province. Row (7) uses a different $\mathrm{SAP}$ indicator, in which $\mathrm{SAP}=1$ when the mobility within a city is restricted. Row (8) uses log deaths as outcome variables, and regression is weighted using population. Here, we use estimate impacts' change in levels using a mean value of each variable. The number of observations is 56,056 (row 3), 59,290 (5), 57,134 (6), and 58,996 (others). The data covers the period from January 1 to April 7, and the standard errors are clustered at the DSP level. We describe the detail in Supplementary Note 2. 

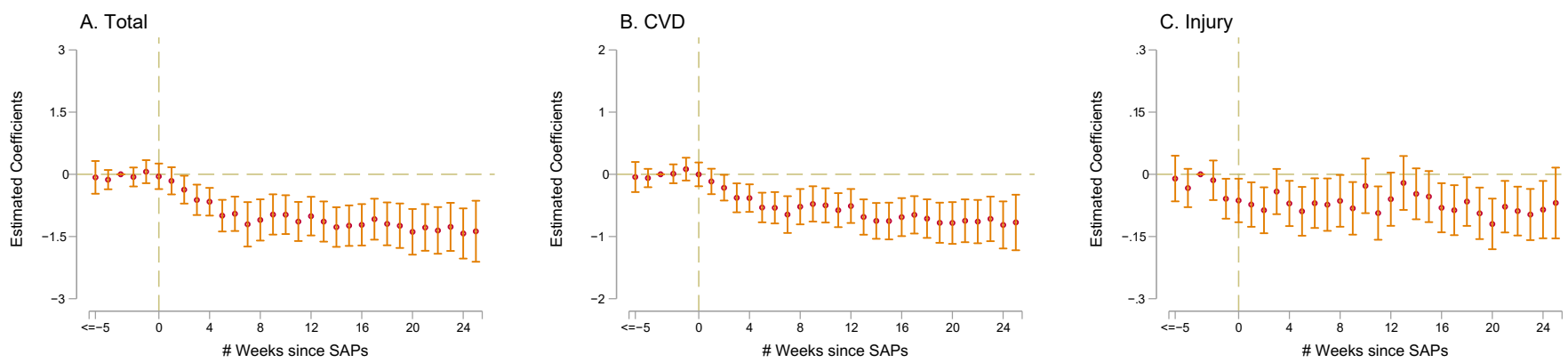

D. Acute Lower Respiratory Infection

E. Chronic Lower Respiratory Infection

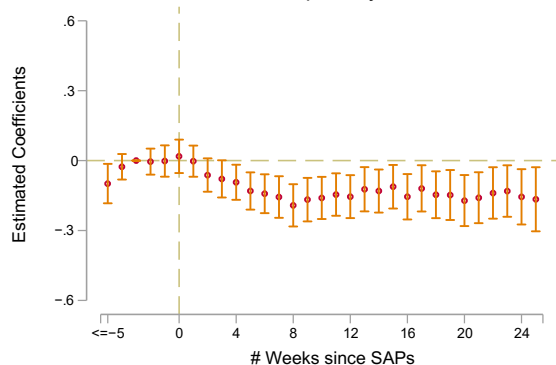

F. Neoplasms

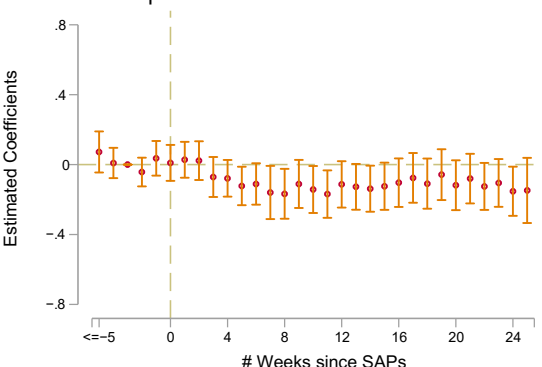

Extended Data Fig. 5 | The event-study results on the short- and medium-term effects of SAPs on non-COVID-19 deaths. Each figure represents a separate regression using the event-study approach (Equation 3). Results use deaths (except for deaths from COVID-19) from all-causes (Panel A), CVD (B), injury (C), ALRI (D), CLRI (E), and neoplasms (F). We include leads and lags of the start of SAPs in the regressions. The dummy variable indicating the week three weeks prior to the SAPs $(k=-3)$ is omitted from the regressions. The estimated coefficients and their $95 \%$ confidence intervals are plotted. The vertical lines refer to the week when SAPs are issued. The number of observations for each regression is 128,226, covering 602 DSPs (3 DSPs in Wuhan are not included). We use mortality data from January 1 to July 31, 2020. DSP fixed effect and date fixed effect are included in each regression, and the standard errors are clustered at the DSP level. 


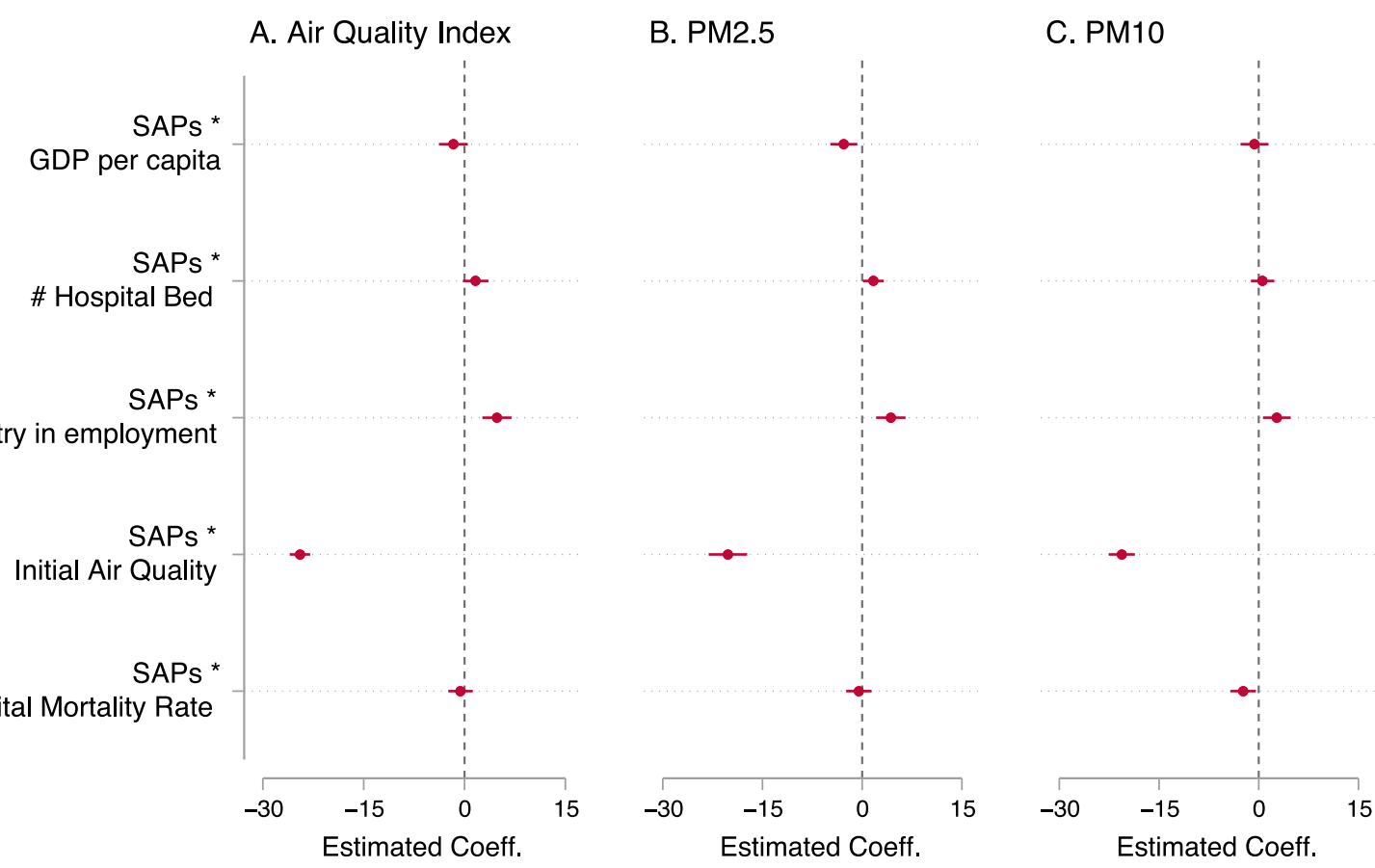

Extended Data Fig. 6 | The heterogeneous impacts of SAPs on air quality. Each column represents a separate DiD regression with plots and bars representing the point estimates and $95 \%$ confidence intervals of interaction terms between the SAP status and the heterogeneous dimensions (Equation 4). The outcome variables are Air Quality Index (Panel A), $\mathrm{PM}_{2.5}(\mathrm{~B})$, and $\mathrm{PM}_{10}(\mathrm{C})$. We use per capita employment, the number of hospital beds per 1,000, the share of secondary industry in GDP, initial Air Quality Index, and initial mortality rate as heterogeneous dimensions. The details of these data are described in Methods. These variables are standardized to have a mean 0 and standard deviation of 1 so that the coefficients of interaction terms are comparable across variables. The number of observations for each regression is 58,996 (A), 56,888 (B), and 56,852 (C), covering 602 DSPs (3 DSPs in Wuhan are not included). We use air quality data from January 1 to April 7, 2020. DSP fixed effect and date fixed effect are included in each regression, and the standard errors are clustered at the DSP level. 
A. Total

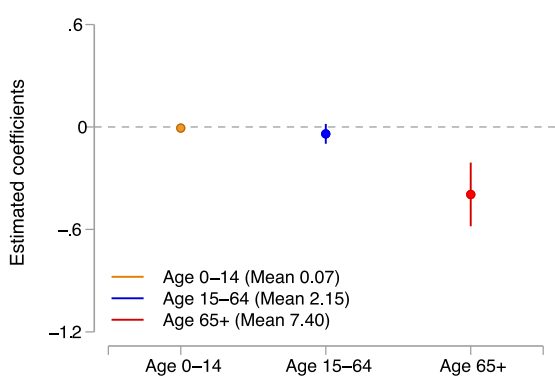

D. Acute Lower Respiratory Infection

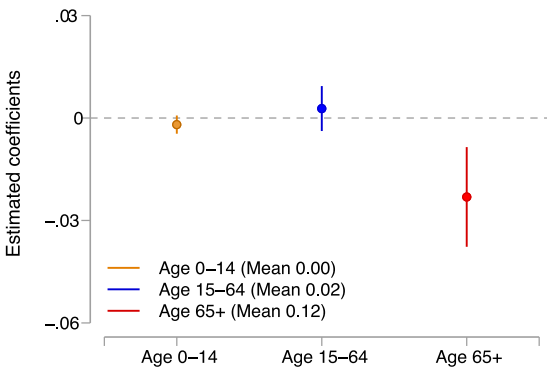

B. CVD

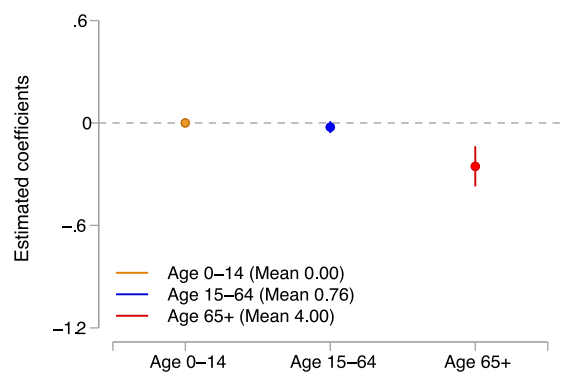

E. Chronic Lower Respiratory Infection

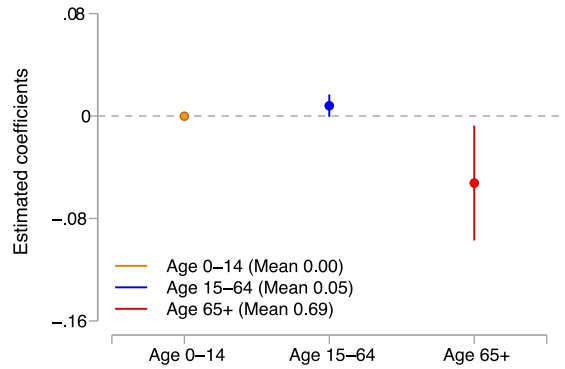

C. Injury

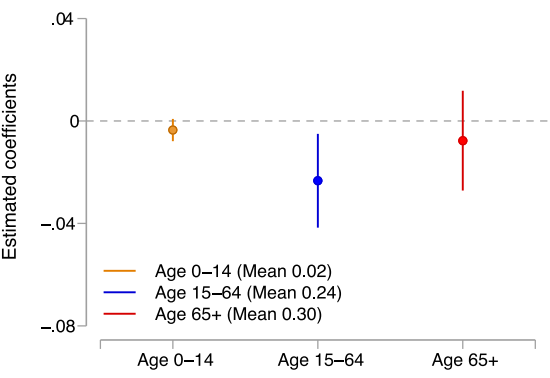

F. Neoplasms

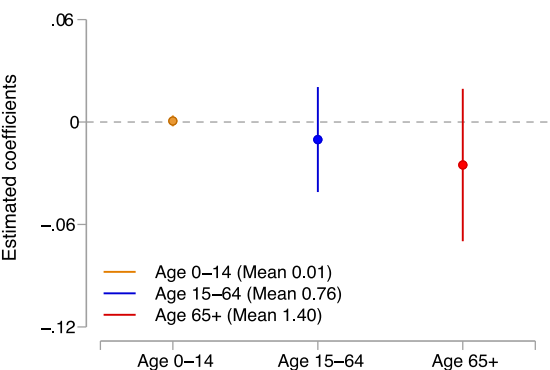

Extended Data Fig. 7 | The impacts of SAPs on deaths by age group. Each plot and bar in the figures represents a separate DiD regression (Equation 1) with dots and the lines representing the point estimates and their $95 \%$ confidence intervals. Results report the effects of SAPs on the number of deaths (except for deaths from COVID-19) from all-causes (Panel A), CVD (B), injury (C), ALRI (D), CLRI (E), and neoplasms (F) for those aged below 15 (orange), aged 15-64 (blue), and aged over 65 (red). DSP fixed effect and date fixed effect are included in all the regressions. The number of observations for each regression is 58,996, covering 602 DSPs (3 DSPs in Wuhan are not included). We use death data from January 1 to April 7, 2020. DSP fixed effect and date fixed effect are included in each regression, and the standard errors are clustered at the DSP level. 


\section{Reporting Summary}

Nature Research wishes to improve the reproducibility of the work that we publish. This form provides structure for consistency and transparency in reporting. For further information on Nature Research policies, see our Editorial Policies and the Editorial Policy Checklist.

\section{Statistics}

For all statistical analyses, confirm that the following items are present in the figure legend, table legend, main text, or Methods section.

n/a Confirmed

$\bigotimes$ The exact sample size $(n)$ for each experimental group/condition, given as a discrete number and unit of measurement

$\square$ A statement on whether measurements were taken from distinct samples or whether the same sample was measured repeatedly

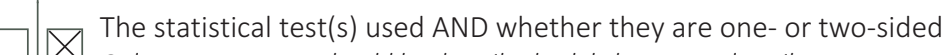

$\square$ Only common tests should be described solely by name; describe more complex techniques in the Methods section.

$\square$ A description of all covariates tested

$\square$ \A description of any assumptions or corrections, such as tests of normality and adjustment for multiple comparisons

A full description of the statistical parameters including central tendency (e.g. means) or other basic estimates (e.g. regression coefficient)

$\triangle$ AND variation (e.g. standard deviation) or associated estimates of uncertainty (e.g. confidence intervals)

$\varnothing$ For null hypothesis testing, the test statistic (e.g. $F, t, r$ ) with confidence intervals, effect sizes, degrees of freedom and $P$ value noted

Give P values as exact values whenever suitable.

Х $\square$ For Bayesian analysis, information on the choice of priors and Markov chain Monte Carlo settings

$\square$ \ For hierarchical and complex designs, identification of the appropriate level for tests and full reporting of outcomes

$\square \bigotimes$ Estimates of effect sizes (e.g. Cohen's $d$, Pearson's $r$ ), indicating how they were calculated

Our web collection on statistics for biologists contains articles on many of the points above.

\section{Software and code}

Policy information about availability of computer code

Data collection Computer codes are not used to collect data

Data analysis Stata $15 \mathrm{MP}$, and R version 3.5 are used to analyze the data . Codes used in this paper are available at https://github.com/yhecon/ Mortality_Covid_China.

For manuscripts utilizing custom algorithms or software that are central to the research but not yet described in published literature, software must be made available to editors and reviewers. We strongly encourage code deposition in a community repository (e.g. GitHub). See the Nature Research guidelines for submitting code \& software for further information,

\section{Data}

Policy information about availability of data

All manuscripts must include a data availability statement. This statement should provide the following information, where applicable:

- Accession codes, unique identifiers, or web links for publicly available datasets

- A list of figures that have associated raw data

- A description of any restrictions on data availability

Data used in this paper are available at https://github.com/yhecon/Mortality_Covid_China. 


\section{Field-specific reporting}

Please select the one below that is the best fit for your research. If you are not sure, read the appropriate sections before making your selection.
Life sciences
Behavioural \& social sciences
Ecological, evolutionary \& environmental sciences

For a reference copy of the document with all sections, see nature.com/documents/nr-reporting-summary-flat.pdf

\section{Behavioural \& social sciences study design}

All studies must disclose on these points even when the disclosure is negative.

Study description

Research sample

Sampling strategy

Data collection

Timing

Data exclusions

Non-participation

Randomization
This study analyzes the short- and medium-term impacts of strict anti-contagion policies on Non-COVID-19 Mortality in China We use death records data from China's Disease Surveillance Points (DSPs) system

The empirical analysis uses the comprehensive death records from China's Disease Surveillance Points (DSPs) system, covering more than 324 million people in 605 DSP's districts/counties in 321 cities. The DSPs were chosen using an iterative method involving multistage stratification and takes into account the sociodemographic characteristics of the population. The DSPs data are representative both at the provincial and national levels. Matching the DSPs data with data on city-level strict anti-contagion policies, we analyze the short- and medium- term impacts of SAPs on non-COVID mortality using a difference-in-differences (DiD) approach.

Weekly mortality data are obtained from the Disease Surveillance Points (DSPs) system managed by the Chinese Center for Disease Control and Prevention. Information on local governments' strict anti-contagion policies (i.e., lockdowns) was collected city by city from news media and government announcements. The weather data are obtained from the China Meteorology Administration (CMA). Air pollution data are collected from the Ministry of Ecology and Environment. Socio-economic data at the city or county level are collected from the 2018 China City Statistical Yearbook and 2018 China County Statistical Yearbook.

Data collection began on January 1, 2020 and end on July 31, 2020.

We exclude three DSPs in Wuhan from the baseline analysis because the city is the epicenter of the outbreak in China. We are concerned that its death reporting process was partially disrupted due to insufficient medical staff supply during the COVID-19 outbreak.

No participants dropped out or declined participation because this study does not directly involve individual participation.

\section{Reporting for specific materials, systems and methods}

We require information from authors about some types of materials, experimental systems and methods used in many studies. Here, indicate whether each material, system or method listed is relevant to your study. If you are not sure if a list item applies to your research, read the appropriate section before selecting a response.

\begin{tabular}{|c|c|}
\hline n/a & Involved in the study \\
\hline$\bigotimes$ & $\square$ Antibodies \\
\hline Х & Eukaryotic cell lines \\
\hline Х & Palaeontology and archaeology \\
\hline$凶$ & Animals and other organisms \\
\hline$\bigotimes$ & $\square$ Human research participants \\
\hline$\bigotimes$ & $\square$ Clinical data \\
\hline$\bigotimes$ & $\square$ Dual use research of concern \\
\hline
\end{tabular}

\begin{tabular}{l|l}
\multicolumn{2}{l}{ Methods } \\
\hline n/a & Involved in the study \\
$\square$ & $\square$ ChIP-seq \\
$\square$ & $\square$ Flow cytometry \\
$\square$ & $\square$ MRI-based neuroimaging
\end{tabular}

